\title{
Geometry of the theta divisor of a compactified jacobian
}

\author{
Lucia Caporaso
}

\section{Contents}

1. Introduction

1.1. Notation and Conventions

1.2. Brill-Noether varieties and Abel maps

1.3. Stability and semistability

2. Technical groundwork

2.1. Basic estimates

2.2. Basic cases

2.3. Divisors imposing independent conditions

3. Irreducibility and dimension

3.1. Irreducible components of the Theta divisor

3.2. Dimension of the image of the Abel map

4. Compactifying the theta divisor

4.1. The compactified Picard variety

4.2. The theta divisor of the compactified jacobian.

References

\section{INTRODUCTION}

The object of this paper is the theta divisor of the compactified Jacobian of a nodal curve; we compute its irreducible components and give it a geometric interpretation.

Let $X$ be a curve of arithmetic genus $g$ and $\operatorname{Pic}^{d} X$ its degree- $d$ Picard variety, parametrizing line bundles of degree $d$. If $X$ is smooth $\operatorname{Pic}^{d} X$ is compact and it is endowed with a principal polarization: the theta divisor; in case $d=g-1$ the theta divisor can be intrinsically defined as the locus of $L \in \mathrm{Pic}^{g-1} X$ such that $h^{0}(X, L) \neq 0$.

If $X$ is singular, $\operatorname{Pic}^{d} X$ fails to be projective, so one needs to replace it with some projective analogue, the so-called "compactified jacobian", or "compactified Picard variety". We shall assume that $X$ is reduced, possibly reducible, and has at most nodes as singularities. Although in the literature there exist several different constructions of compactified jacobians, recent work of V. Alexeev shows that in case $d=g-1$, there exists a "canonical" one. More precisely, in [A104] the compactifications of [OS79], S94] and [C94] are shown to be isomorphic if $d=g-1$, and to behave consistently with the degeneration theory of principally polarized abelian varieties, as we will explain below.

These recent developements revive interest in the theory of Brill-Noether varieties for singular curves, of which the theta divisor is one of the principal instances.

Basic results on the theta divisor of the generalized jacobian of a (reducible) nodal curve were proved by A. Beauville in [B77]. Later on, the theta divisor was extended to the compactified jacobian of an integral curve 
by A. Soucaris, S94, E. Esteves [E97] and, more recently, for a reducible nodal curve by V. Alexeev Al04. Thanks to the work of these mathematicians, we know that in degree $(g-1)$ the compactified Picard variety of any nodal curve $X$ has a theta divisor $\Theta(X)$ which is Cartier, ample, and provides a polarization such that the pair (Compactified Jacobian, Theta Divisor ) is a semiabelic stable pair in the sense of [Al02]. Furthermore, the above holds in the relative setting, i.e. for families of nodal curves.

The purpose of this paper is to investigate of the geometry of $\Theta(X)$ more closely and to understand its modular meaning. The first main result (Theorem 3.2) describes its irreducible components, showing that every irreducible component of the compactified jacobian contains a unique irreducible component of the theta divisor. In more technical terms, this is the same as saying that for every fixed "stable" multidegree (see Definition 1.3.1) the theta divisor has a unique irreducible component. We wish to point out that this result is sharp: as Examples 3.4 and 3.5 show, irreducibility fails for non stable multidegrees.

We prove Theorem 3.2 by viewing it in connection with the Abel map. As a by-product, we obtain that the theta divisor is the closure of the image of the Abel maps for every stable multidegree.

In the second part of the paper we give an explicit geometric description of $\Theta(X)$ in terms of moduli theory. We do that in Theorem 4.5, exhibiting a stratification by means of the theta divisors of the partial normalizations of $X$. This yields a modular description in terms of effective line bundles on the partial normalizations of $X$, or (which turns out to be the same) in terms of line bundles on semistable curves stably equivalent to $X$.

The paper consists of four sections. The first contains preliminaries and basic definitions; the second is mostly made of technical results. In the third section we prove the irreducibility theorem and study the dimension of the image of the Abel map (Proposition 3.6). In the fourth section we describe the compactification of the theta divisor inside the compactified jacobian.

\subsection{Notation and Conventions.}

1.1.1. We work over an algebraically closed field $k$. By the word "curve" we mean a reduced, projective curve over $k$.

Throughout the paper $X$ will be a connected nodal curve of arithmetic genus $g$, having $\gamma$ irreducible components and $\delta$ nodes. We call $\nu: Y \rightarrow X$ the normalization of $X$, so that $Y=\coprod_{i=1}^{\gamma} C_{i}$ with $C_{i}$ is smooth of genus $g_{i}$, and $X=\cup \overline{C_{i}}$ with $\overline{C_{i}}=\nu\left(C_{i}\right)$. Recall that

$$
g=\sum_{i=1}^{\gamma} g_{i}+\delta-\gamma+1
$$

Observe that the above formula holds regardless of $X$ being connected.

More notation: let $S \subset X_{\text {sing }}$ be a set of nodes of $X$, set $\# S:=\delta_{S}$ and $S=\left\{n_{1}, \ldots, n_{\delta_{S}}\right\}$. The (partial) normalization of $X$ at the nodes in $S$ will be denoted $\nu_{S}: Y_{S} \longrightarrow X$ and $\gamma_{S}$ will be the number of connected components of $Y_{S}$, so that $Y_{S}=\coprod_{1}^{\gamma_{S}} Y_{i}$ with $Y_{i}$ a connected curve of arithmetic genus 
$g_{Y_{i}}$. From (1) we get

$$
g=\sum_{i=1}^{\gamma_{S}} g_{Y_{i}}+\delta_{S}-\gamma_{S}+1
$$

and, denoting $g_{Y_{S}}=p_{a}\left(Y_{S}\right)$

$$
g_{Y_{S}}=g-\delta_{S}=\sum_{i=1}^{\gamma_{S}} g_{Y_{i}}-\gamma_{S}+1
$$

For every $j=1 \ldots, \delta_{S}$ (or for every $n \in S$ ) we set

$$
\nu_{S}^{-1}\left(n_{j}\right)=\left\{q_{1}^{j}, q_{2}^{j}\right\} \quad\left(\text { or } \nu_{S}^{-1}(n)=\left\{q_{1}, q_{2}\right\}\right) .
$$

1.1.2. To a nodal curve $Y$ one associates its dual graph $\Gamma_{Y}$, having as vertices the irreducible components of $Y$, as edges the nodes of $Y$. A node lying in a unique irreducible component $C_{i}$ of $Y$ corresponds to a loop of $\Gamma_{Y}$ based at the vertex $C_{i}$; a node lying in $C_{i} \cap C_{j}$ corresponds to an edge joining the vertices $C_{i}$ and $C_{j}$.

1.1.3. The degree- $d$ Picard variety $\operatorname{Pic}^{d} X$ has a decomposition into connected/irreducible components: $\operatorname{Pic}^{d} X=\coprod_{\underline{d} \in \mathbb{Z}^{\gamma}:|\underline{d}|=d} \operatorname{Pic}^{\underline{d}} X$, where Pic $\underline{d} X$ is the variety of isomorphism classes of line bundles of multidegree $\underline{d}$.

Let $\nu_{S}: Y_{S} \longrightarrow X$ be as in 1.1.1, Consider the pull-back map

$$
\operatorname{Pic} X \stackrel{\nu_{S}^{*}}{\longrightarrow} \operatorname{Pic} Y_{S} \cong \prod_{i=1}^{\gamma_{S}} \operatorname{Pic} Y_{i} \longrightarrow 0
$$

We shall usually identify $\operatorname{Pic} Y_{S} \cong \prod \operatorname{Pic} Y_{i}$ without mentioning it.

Let $M \in \operatorname{Pic} Y_{S}$, then the fiber over $M$ will be denoted

$$
F_{M}(X):=\left\{L \in \operatorname{Pic} X: \nu_{S}^{*} L=M\right\} \cong\left(k^{*}\right)^{\delta_{S}-\gamma_{S}+1}
$$

1.1.4. We shall now describe the isomorphism $F_{M}(X) \cong\left(k^{*}\right)^{\delta_{S}-\gamma_{S}+1}$ explicitely to fix some conventions. Let us simplify the notation by omitting the subscript $S$ (so, $\delta=\delta_{S}, Y=Y_{S} \ldots$ ). Assume first that $Y$ is connected.

Let $\underline{c}=\left(c_{1}, \ldots, c_{\delta}\right) \in\left(k^{*}\right)^{\delta} ; \underline{c}$ determines a unique $L \in \operatorname{Pic} X$ such that $\nu^{*} L=M$ as follows. For every $j=1, \ldots, \delta$ consider the two fibers of $M$ over $q_{1}^{j}$ and $q_{2}^{j}$ (recall that $\nu\left(q_{1}^{j}\right)=\nu\left(q_{2}^{j}\right)=n_{j}$ ). We define a line bundle $L=L^{(\underline{c})}$ on $X$ which pulls back to $M$, by gluing $M_{q_{1}^{j}}$ to $M_{q_{2}^{j}}$ via the isomorphism

$$
M_{q_{1}^{j}} \stackrel{\cdot c_{j}}{\longrightarrow} M_{q_{2}^{j}}
$$

given by multiplication by $c_{j}$. Conversely, every $L \in F_{M}(X)$ is of type $L^{(\underline{c})}$.

Now let $Y$ have $\gamma$ connected components; note that, since $X$ is connected, we always have $\gamma-1 \leq \delta$. There exist some subsets $T \subset S$ such that $\# T=\gamma-1$ and such that if we remove from $\Gamma_{X}$ every node that is not in $T$, the remaining graph is a connected tree (a so-called spanning tree of $\Gamma_{X}$ ).

Let us fix one such $T$ and order the nodes in $S$ so that the last $\gamma-1$ are in $T$, i.e. $S=\left\{n_{1}, \ldots, n_{\delta}\right\}=\left\{n_{1}, \ldots, n_{\delta-\gamma+1}\right\} \cup T$. Now factor $\nu$ as follows

$$
\nu: Y \stackrel{\nu_{T}}{\longrightarrow} Y^{\prime} \stackrel{\nu^{\prime}}{\longrightarrow} X
$$


so that $\nu^{\prime}$ is the partial normalization of $X$ at $S \backslash T$ and $\nu_{T}$ the normalization at the nodes of $Y^{\prime}$ preimages of the nodes in $T$. For example, if $S=X_{\text {sing }}$ (i.e. if $Y$ is smooth) then $Y^{\prime}$ is a curve of compact type. The pull-back map $\nu_{T}^{*}$ induces an isomorphism $\operatorname{Pic} Y^{\prime} \cong \operatorname{Pic} Y$, i.e. different gluing data determine isomorphic line bundles on $Y^{\prime}$.

Now, to construct the fiber of $\operatorname{Pic} X \rightarrow \operatorname{Pic} Y^{\prime}$ over $M^{\prime}$ we proceed as in the previous part.

Summarizing, for every $\underline{c} \in\left(k^{*}\right)^{\delta-\gamma+1}$ we associate a unique $L^{(\underline{c})} \in \operatorname{Pic} Y$; since the gluing data over the nodes in $T$ is irrelevant, we shall fix $c_{j}=1$ if $j \geq \delta-\gamma$ and use that as gluing constant over $T$.

Finally observe that a section $s \in H^{0}(Y, M)$ descends to a section $\bar{s} \in$ $H^{0}\left(X, L^{(\underline{c})}\right)$ if and only if for every $j=1, \ldots, \delta$ we have

$$
s\left(q_{2}^{j}\right)=c_{j} s\left(q_{1}^{j}\right) .
$$

\subsection{Brill-Noether varieties and Abel maps.}

1.2.1. We recall some basic facts about Brill-Noether varieties for smooth curves, following the notation of [ACGH] to which we refer for details.

Let $C$ be a smooth connected curve of genus $g \geq 0$, and let $d$ and $r$ be nonnegative integers. The set $\mathrm{W}_{d}^{r}(C):=\left\{L \in \mathrm{Pic}^{d} C: h^{0}(C, L) \geq r+1\right\}$ has an algebraic structure and is called a Brill-Noether variety. It is closely related to the Abel map in degree $d$ of $C$, that is the map

$$
\begin{aligned}
& \alpha_{C}^{d}: \quad C^{d} \quad \longrightarrow \quad \operatorname{Pic}^{d}(C) \\
& \left(p_{1}, \ldots, p_{d}\right) \quad \mapsto \mathcal{O}_{C}\left(\sum_{1}^{d} p_{i}\right) .
\end{aligned}
$$

Then $\operatorname{Im} \alpha_{C}^{d} \subseteq \mathrm{W}_{d}^{r}(C)$ for all $r \geq 0$ (see 1.2 for when equality occurs). Note that $\mathrm{W}_{d}^{r}(C)$ may fail to be irreducible, so when talking about its dimension we will mean the maximum dimension of its components. The following is well known( ACGH] Lemma 3.3 Ch.IV)

Fact 1.1. If $r \geq d-g$, then $\operatorname{dim} \mathrm{W}_{d}^{r}(C) \geq \rho(g, r, d):=g-(r+1)(r-d+g)$. If $r \leq d-g$ then (by Riemann-Roch) $\mathrm{W}_{d}^{r}(C)=\mathrm{W}_{d}^{d-g}(C)$

There is also a simple upper bound

$$
\operatorname{dim} \mathrm{W}_{d}^{r}(C) \leq \min \{d-r, g\}
$$

Indeed, if $d-r \leq g$, it suffices to look at the Abel map of degree $d$ to obtain that $\operatorname{dim} \mathrm{W}_{d}^{r}(C) \leq d-r$ (cf. ACGH] Prop. 3.4 Ch.IV). If $d-r \geq g$ then, by Riemann-Roch, $\operatorname{dim} \mathrm{W}_{d}^{r}(C)=g$

Remark 1.2. Denote by $r(d)$ the dimension of a general (non empty) complete linear system of degree $d$. i.e. if $d \leq g$ set $r(d)=0$, if $d \geq g$ set $r(d)=$ $d-g$. Note that $\mathrm{W}_{d}^{r(d)}(C)=\operatorname{Im} \alpha_{C}^{d}$. Now, $\min \{d-r(d), g\}=\min \{d, g\}$ and

$$
\operatorname{dim} \mathrm{W}_{d}^{r}(C) \begin{cases}=\min \{d, g\} & \text { if } r \leq r(d) \\ <\min \{d, g\} & \text { if } r>r(d)\end{cases}
$$

To see that, assume first that $r \leq r(d)$, then $\mathrm{W}_{d}^{r}(C)=\mathrm{W}_{d}^{r(d)}(C)$ by Riemann-Roch so we may assume that $r=r(d)$. Now computing gives $\rho(d, g, r(d))=\min \{d, g\}$, so by fact 1.1 and (8) we get $\operatorname{dim} \mathrm{W}_{d}^{r}(C)=$ $\min \{d, g\}$. The case $r>r(d)$ follows from (8) and the fact that $\min \{d-$ $r, g\}<\min \{d-r(d), g\}$. 
1.2.2. For a nodal curve $X$ of genus $g$ having $\gamma$ irreducible components, for any $\underline{d} \in \mathbb{Z}^{\gamma}$ and $r \geq 0$, we set $W_{\underline{d}}^{r}(X):=\left\{L \in \operatorname{Pic} \underline{d} X: h^{0}(X, L) \geq r+1\right\}$ and for any $d \in \mathbb{Z} W_{d}^{r}(X):=\coprod_{|\underline{d}|=d} W_{\underline{d}}^{r}(X)$.

In case $r=0$ we shall usually omit the superscript $r=0$. In particular

$$
\mathrm{W}_{g-1}(X):=\left\{L \in \operatorname{Pic}^{g-1} X: h^{0}(X, L) \geq 1\right\}=\coprod_{|\underline{d}|=g-1} W_{\underline{d}}(X) .
$$

We shall denote (recall (5))

$$
W_{M}(X):=\left\{L \in F_{M}(X): h^{0}(X, L) \geq 1\right\}
$$

Remark 1.3. The above definitions make sense also for non connected curves. Consider a disconnected curve, $Y=\coprod_{i=1}^{\gamma} C_{i}$ where $C_{i}$ is smooth and connected (or more generally $C_{i}$ irreducible) of genus $g_{i}$. For any $\underline{d} \in \mathbb{Z}^{\gamma}$, the variety $\mathrm{W}_{\underline{d}}(Y)$ is easily described in terms of the $C_{i}$ :

$$
\mathrm{W}_{\underline{d}}(Y)= \begin{cases}\prod_{i=1}^{\gamma} \operatorname{Pic}^{d_{i}} C_{i} & \text { if } \exists i: d_{i} \geq g_{i} \\ \bigcup_{j=1}^{\gamma}\left(\mathrm{W}_{d_{j}}\left(C_{j}\right) \times \prod_{i \neq j, i=1, \ldots, \gamma} \operatorname{Pic}^{d_{i}} C_{i}\right) & \text { if } \forall i: d_{i} \leq g_{i}-1\end{cases}
$$

We shall need the following very simple

Lemma 1.4. Let $S \subset X_{\text {sing }}, \nu_{S}: Y_{S} \rightarrow X$ the normalization of $X$ at $S$ and $p \in X \backslash S$. Let $M \in \operatorname{Pic} Y_{S}$ and assume that $M$ has no base point in $\nu_{S}^{-1}(S \cup p)$. Then there exists $L \in W_{M}(X)$ such that $L$ has no base point in $p$. In particular, if $M$ has no base point over $S$ then $W_{M}(X)$ is nonempty.

Proof. To say that $M$ has no base point in $\nu_{S}^{-1}(S \cup p)$ is to say that there exists $s \in H^{0}\left(Y_{S}, M\right)$ such that $s(q) \neq 0$ for every $q \in \nu_{S}^{-1}(S \cup p)$. We can use $s$ to construct a line bundle $L \in W_{M}(X)$ by identifying the two fibers over pairs of corresponding branches. More precisely, with the notation of 1.1.4 (6) for every $n_{j} \in S$ call $q_{1}^{j}, q_{2}^{j}$ the branches over $n_{j}$. Then set $c_{j}:=s\left(q_{2}^{j}\right) / s\left(q_{1}^{j}\right)$ and define $L=L^{(\underline{c})}$. It is clear that $s$ descends to a nonzero section $\bar{s}$ of $L$ and that $\bar{s}(p) \neq 0$.

1.2.3. Abel maps. We now introduce the Abel maps of a singular curve. Recall (see 1.1.1) that $X=\bar{C}_{1} \cup \ldots \cup \bar{C}_{\gamma}$ denotes the decomposition of $X$ into irreducible components. For every $\underline{d}=\left(d_{1}, \ldots, d_{\gamma}\right)$ such that $d_{i} \geq 0$ we set $X^{\underline{d}}:=\bar{C}_{1}^{d_{1}} \times \ldots \times \bar{C}_{\gamma}^{d_{\gamma}}$. Now denote $\dot{X}:=X \backslash X_{\text {sing }}$ the smooth locus of $X$. The normalization map $Y=\cup C_{i} \stackrel{\nu}{\longrightarrow} X=\cup \bar{C}_{i}$ induces an isomorphism of $\dot{X}$ with $Y \backslash \nu^{-1}\left(X_{\text {sing }}\right)$. We shall identify $\dot{X}=Y \backslash \nu^{-1}\left(X_{\text {sing }}\right)$ and denote $\dot{C}_{i}:=\bar{C}_{i} \cap \dot{X}$. Finally, set

$$
\dot{X}^{\underline{d}}:=\dot{C}_{1}^{d_{1}} \times \ldots \times \dot{C}_{\gamma}^{d_{\gamma}} \subset X^{\underline{d}}
$$

so that $\dot{X}^{\underline{d}}$ is a smooth irreducible variety of dimension $|\underline{d}|$, open and dense in $X \underline{d}$. Set $d=|d|$, then we have a regular map

$$
\begin{array}{cccr}
\alpha_{X}^{\frac{d}{X}}: & \dot{X^{\underline{d}}} & \longrightarrow & \operatorname{Pic}^{\underline{d}} X \\
& \left(p_{1}, \ldots, p_{d}\right) & \mapsto & \mathcal{O}_{X}\left(\sum_{1}^{d} p_{i}\right)
\end{array}
$$


which we call the Abel map of multidegree $\underline{d}$. We denote

$$
A_{\underline{d}}(X):=\overline{\alpha^{\frac{d}{X}}(\dot{X} \underline{d})} \subset \operatorname{Pic}^{\underline{d}} X .
$$

Lemma 1.5. Let $X$ be a nodal curve of genus $g \geq 0$. For every $d \geq 1$ and every multidegree $\underline{d}$ on $X$ such that $\underline{d} \geq 0$ and $|\underline{d}|=d$ we have

(i) $A_{\underline{d}}(X)$ is irreducible and $\operatorname{dim} A_{\underline{d}}(X) \leq \min \{d, g\}$;

(ii) $\overline{A_{\underline{d}}}(X) \subset W_{\underline{d}}(X)$

Proof. (1) is obvious, as $X^{\underline{d}}$ is irreducible of dimension $d$ and $\operatorname{dim} \operatorname{Pic}^{\underline{d}} X=g$.

Now, for any $\left(p_{1}, \ldots, p_{d}\right) \in \dot{X}^{\underline{d}}$ we have that $h^{0}\left(X, \sum p_{i}\right) \geq 1$, hence the second statement follows by uppersemicontinuity of $h^{0}$.

We shall see that strict inequality in (ii) does occur (in Proposition 3.6).

1.3. Stability and semistability. As we said in the introduction, there exist various modular descriptions for a compactified Picard variety, and they are equivalent if $d=g-1$ (by [Al04]). In particular, two different equivalent definitions of semistability and stability are available (1.3.1 and 1.3.2 below); the simultaneous use of the two turns out to be a good tool to overcome technical difficulties of combinatorial type.

1.3.1. Stability: Definition 1. Let $Y$ be a nodal curve of arithmetic genus $g$ having $\gamma$ irreducible components. Let $\underline{d} \in \mathbb{Z}^{\gamma}$ be such that $|\underline{d}|=g-1$.

(a) We call $\underline{d}$ semistabl $\oplus^{1}$ if for every subcurve (equivalently, every connected subcurve) $Z \subset Y$ of arithmetic genus $g_{Z}$ we have

$$
d_{Z} \geq g_{Z}-1
$$

where $d_{Z}:=\left|\underline{d}_{Z}\right|$. The set of semistable multidegrees on $Y$ is denoted

$$
\Sigma^{s s}(Y):=\left\{\underline{d} \in \mathbb{Z}^{\gamma}:|\underline{d}|=g-1, \underline{d} \text { is semistable }\right\}
$$

(b) Assume $Y$ connected. If $Y$ is irreducible, or if strict inequality holds in (11) for every (connected) subcurve $Z \subsetneq Y$, then $\underline{d}$ is called stable 2 .

If $Y$ is not connected, we say that $\underline{d}$ is stable if its restriction to every connected component of $Y$ is stable. We denote

$$
\Sigma(Y):=\left\{\underline{d} \in \mathbb{Z}^{\gamma}:|\underline{d}|=g-1, \underline{d} \text { is stable }\right\} \subset \Sigma^{s s}(Y)
$$

We shall also use the following equivalent definition, originating from [B77].

\subsubsection{Stability: Definition 2. Let $Y$ and $\underline{d}$ as in 1.3.1.}

(A) $\underline{d}$ is semistable if the dual graph $\Gamma_{Y}$ of $Y$ (cf. 1.1.2) can be oriented in such a way that, denoting by $b_{i}$ the number of edges pointing at the vertex corresponding to the irreducible component $C_{i}$ of $Y$, then

$$
d_{i}=g_{i}-1+b_{i}
$$

where $g_{i}$ is the geometric genus of $C_{i}$ (so that $g_{i}=p_{a}\left(C_{i}\right)-\#\left(C_{i}\right)_{\operatorname{sing}}$ ).

\footnotetext{
${ }^{1}$ Semistable is the same as balanced in $\mathrm{C} 05$.

${ }^{2}$ Stable here is not the same as stably balanced in $\mathrm{C} 05$.
} 
(B) Assume $Y$ connected. Then $\underline{d}$ is stable if $\Gamma_{Y}$ admits an orientation satisfying (A) and such that there exists no proper subcurve $Z \subsetneq Y$ such that the edges between $\Gamma_{Z}$ and $\Gamma_{Z^{C}}$ go all in the same direction direction, where $Z^{C}:=\overline{Y \backslash Z}$.

The equivalence of definitions 1.3.2 and 1.3.1 is Prop.3.6 in Al04. The version given in 1.3.2 (A) is due to A.Beauville, who used it in [B77] to define and study the theta divisor of a generalized jacobian (Note that in [B77] Lemma (2.1) the dual graph is without loops by definition, whereas we need to include loops. This explains the formal difference between our definition and that of [B77]).

Version 1.3 .1 actually extends to all degrees (other than degree $g-1$ ); it originates from D.Gieseker's construction of $\bar{M}_{g}$ and is crucial in the construction of the compatified Picard variety in [C94] (where (11) is generalized by the so-called "Basic Inequality"). V.Alexeev proved that the Basic Inequality yields the modular description of the compactified jacobians constructed by Oda-Seshadri and by C.Simpson using different approaches (see Al04 $1.7(5)$ ). More details about this definition and its connection with Geometric Invariant Theory will be given in Section 4 .

Remark 1.6. (i) Applying inequality (11) to all subcurves, we get that $\underline{d}$ is semistable if and only if for every connected $Z \subset Y$

$$
p_{a}(Z)-1 \leq d_{Z} \leq p_{a}(Z)-1+\# Z \cap Z^{C} .
$$

If $X$ is connected, $\underline{d}$ is stable if and only if strict inequalities hold in (12) for all $Z$.

(ii) If $\underline{d} \in \Sigma^{s s}(X)$ and $V \subset X$ is a subcurve such that $d_{V}=g_{V}-1$, then $\underline{d}_{V}$ is semistable on $V$.

(iii) If $\underline{d}$ is stable, then $\underline{d} \geq 0$.

Remark 1.7. The following convention turns out to be useful. Given a graph $\Gamma$ (e.g. $\Gamma=\Gamma_{Y}$ ), every edge $n$ determines two half-edges, denoted $q_{1}^{n}$ and $q_{2}^{n}$ (corresponding to the two branches of the node $n$ of $Y$ ). If $\Gamma$ is oriented we call $q_{1}^{n}$ the starting half-edge of $n$ and $q_{2}^{n}$ the ending one.

$\Sigma^{s s}(X)$ is never empty (by [C05] Prop. 4.2). On the other hand we have

Lemma 1.8. Let $X$ be a connected curve. $\Sigma(X)=\emptyset$ if and only if $X$ has a separating node.

Proof. If $X$ has a separating node, $n$, then $X=X_{1} \cup X_{2}$ with $X_{1} \cap X_{2}=\{n\}$. Let $\underline{d} \in \Sigma^{s s}(X)$, using (12) we have

$$
p_{a}\left(X_{i}\right)-1 \leq d_{X_{i}} \leq p_{a}\left(X_{i}\right)
$$

so that, obviously, strict inequalities cannot simultaneously occur. Hence $\underline{d}$ is not stable.

Conversely, assume that $X$ has no separating node. We shall use Definition 1.3.2, and prove that the dual graph of $X, \Gamma=\Gamma_{X}$, admits a "stable orientation" (i.e. an orientation satisfying $(\mathrm{B})$ ). We use induction on the number $\delta$ of nodes that lie in two different irreducible components (the only nodes that matter), i.e. induction on the number of edges that are not loops. If $\delta=1$ there is nothing to prove (the edge is necessarily separating), if $\delta=2$ then $\Gamma$ has two vertices so the statement is clear. 
Let $\delta \geq 2$, pick and edge $n$ and let $\Gamma^{\prime}=\Gamma-n$; thus $\Gamma^{\prime}$ is connected. If $\Gamma^{\prime}$ has no separating edge, by induction $\Gamma^{\prime}$ admits a stable orientation, hence so does $\Gamma$, of course. Denote $n_{1}, \ldots, n_{t}$ the separating edges of $\Gamma^{\prime}$. The graph

$$
\Gamma^{\prime}-\left\{n_{1}, \ldots, n_{t}\right\}=\Gamma-\left\{n_{0}, n_{1}, \ldots, n_{t}\right\}
$$

where $n=n_{0}$, has $t+1$ connected components, $\overline{\Gamma_{0}}, \ldots, \overline{\Gamma_{t}}$, each of which is free from separating edges.

We claim that the image $\Gamma_{i} \subset \Gamma$ of each $\overline{\Gamma_{i}}$ contains exactly two of the edges $n_{0}, n_{1}, \ldots, n_{t}$.

Indeed, if (say) $\Gamma_{1}$ contains only one $n_{i}$ with $i \geq 1$, call it $n_{1}$ and call $\Gamma_{2}$ the other $\Gamma_{i}$ containing $n_{1}$. Then $n_{0}$ connects $\Gamma_{1}$ with $\Gamma_{2}$ (for otherwise $n_{1}$ would be a separating node of $\Gamma$ which is not possible). Hence $\Gamma_{1}$ contains $n_{0}$ and $n_{1}$.

If $\Gamma_{1}$ contains two $n_{i}$ with $i \geq 1$, call them $n_{1}$ and $n_{2}$, let $\Gamma_{2}$ and $\Gamma_{3}$ be such that $n_{i} \in \Gamma_{1} \cap \Gamma_{i+1}, i=1,2$. Then $n_{0}$ connects $\Gamma_{2}$ and $\Gamma_{3}$, thus $n_{0} \notin \Gamma_{1}$. Therefore $\Gamma_{1}$ contains only $n_{1}$ and $n_{2}$.

If $\Gamma_{1}$ contains three $n_{i}, i \geq 1$, call them $n_{1}, n_{2}$ and $n_{3}$, let $\Gamma_{2}, \Gamma_{3}$ and $\Gamma_{4}$ be such that $n_{i} \in \Gamma_{1} \cap \Gamma_{i+1}$. Now $n_{0}$ is contained in at most two $\Gamma_{i}$, so say $n_{0} \notin \Gamma_{4}$ (say), but then $n_{3}$ is a separating node of $\Gamma$, which is a contradiction. Therefore, up to reordering the $\Gamma_{i}$, we can assume that

$$
n_{i} \in \Gamma_{i} \cap \Gamma_{i-1}, \quad i=1, \ldots, t, t+1=0 .
$$

We now define an orientation on $\Gamma$ by combining the stable orientation on each $\Gamma_{i}$ with each edge $n_{i}$ oriented from $\Gamma_{i-1}$ to $\Gamma_{i}$. It suffices to prove that this is a stable orientation on $\Gamma$.

Indeed: let $Z \subset X$ and $\Gamma_{Z} \subset \Gamma$ the corresponding graph. If for some $i$ we have $\emptyset \neq \Gamma_{Z} \cap \Gamma_{i} \subsetneq \Gamma_{i}$, then inside $\Gamma_{i}$ there are edges both starting from and ending in $\Gamma_{Z}$. So the same holds in $\Gamma$ and we are done. Hence we can assume that for every $i$ either $\Gamma_{i} \subset \Gamma_{Z}$ or $\Gamma_{Z} \cap \Gamma_{i}=\emptyset$. Therefore

$$
\Gamma_{Z} \cap \Gamma_{Z^{C}} \subset\left\{n_{0}, n_{1}, \ldots, n_{t}\right\} .
$$

We can thus reduce ourselves to consider the graph obtained by contracting every $\Gamma_{i}$ to a point. This is of course a cyclic graph with $t+1$ vertices and $t+1$ edges $\left\{n_{0}, n_{1}, \ldots, n_{t}\right\}$, oriented cyclically. This is a stable orientation, so we are done.

Example 1.9. Let $X$ be a nodal connected curve of genus $g, X_{\text {sep }} \subset X_{\text {sing }}$ the set of its separating nodes and $\widetilde{X} \rightarrow X$ the normalization of $X$ at $X_{\mathrm{sep}}$. Assume $\# X_{\text {sep }}=c-1 \geq 1$ so that $\widetilde{X}$ has $c$ connected components $X_{1}, \ldots, X_{c}$ and $X_{i}$ is free from separating nodes for every $i=1, \ldots, c$. Thus $\Sigma\left(X_{i}\right) \neq \emptyset$ and

$$
\Sigma(\widetilde{X})=\Sigma\left(X_{1}\right) \times \cdots \times \Sigma\left(X_{c}\right) .
$$

Indeed, set $g_{i}:=p_{a}\left(X_{i}\right)$, then $p_{a}(\tilde{X})-1=(g-c+1)-1=\sum_{i=1}^{c}\left(g_{i}-1\right)$, and $\underline{d} \in \Sigma(\widetilde{X})$ if and only the restriction $\underline{d}_{i}$ of $\underline{d}$ to $X_{i}$ is stable on $X_{i}$.

Proposition 1.10 (Beauville). Let $X$ be a connected nodal curve of genus $g \geq 1$, and let $\underline{d} \in \mathbb{Z}^{\gamma}$ be such that $|\underline{d}|=g-1$ (same notation as 1.3.1).

(i) $\underline{d}$ is semistable iff there exists $L \in \mathrm{Pic}^{\underline{d}} X$ such that $h^{0}(X, L)=0$. 
(ii) If $\underline{d}$ is semistable then every irreducible component of $W_{\underline{d}}(X)$ has dimension $g-1$.

(iii) If $\underline{d}$ is not semistable then $W_{\underline{d}}(X)=\operatorname{Pic} \underline{\underline{d}} X$.

See Lemma (2.1) and Proposition 2.2 in B77.

1.3.3. Our first main result (Theorem 3.2) states that, if $\underline{d}$ is stable, then $W_{d}(X)$ is irreducible and equal to $A_{d}(X)$. The strategy of the proof is the following. We know, by the above Proposition 1.10, that every irreducible component of $W_{\underline{d}}(X)$ has dimension $g-1$; we also know that $A_{\underline{d}}(X)$ is irreducible. We shall prove that if $W$ is an irreducible component of $W_{\underline{d}}(X)$ not dominated by the image of the Abel map, then $\operatorname{dim} W \leq g-2$, and hence $W$ must be empty. Before doing that, we shall carry out some technical work.

\section{TECHNICAL GROUNDWORK}

2.1. Basic estimates. Recall the set-up of 1.1.1.

Proposition 2.1. Fix $\nu_{S}: Y_{S} \longrightarrow X$ and let $M \in \operatorname{Pic} Y_{S}$.

(i) For every $L \in \operatorname{Pic} X$ such that $\nu_{S}^{*} L=M$ we have

$$
h^{0}\left(Y_{S}, M\right)-\delta_{S} \leq h^{0}(X, L) \leq h^{0}\left(Y_{S}, M\right) .
$$

(ii) Let $h^{0}\left(Y_{S}, M\right) \geq \delta_{S}$. Assume that for some $h:\left\{1, \ldots, \delta_{S}\right\} \rightarrow\{1,2\}$,

$$
h^{0}\left(Y_{S}, M\left(-\sum_{j=1}^{\delta_{S}} q_{h(j)}^{j}\right)\right)=h^{0}(M)-\delta_{S} .
$$

Then $W_{M}(X)$ is of pure dimension:

$$
\operatorname{dim} W_{M}(X)= \begin{cases}\delta_{S}-\gamma_{S} & \text { if } h^{0}(M)=\delta_{S} \\ \delta_{S}-\gamma_{S}+1 & \text { if } h^{0}(M) \geq \delta_{S}+1\end{cases}
$$

Moreover, the general element $L \in W_{M}(X)$ satisfies

$$
h^{0}(X, L)=\max \left\{h^{0}\left(Y_{S}, M\right)-\delta_{S}, 1\right\} .
$$

Proof. Throughout the proof we shall simplify the notation by forgetting the index $S$, i.e. set $Y=Y_{S}, \delta=\delta_{S}, \nu=\nu_{S}$ and $\gamma=\gamma_{S}$.

Let $L \in F_{M}(X)$, then we have the exact sequence

$$
0 \longrightarrow L \longrightarrow \nu_{*} M \longrightarrow \sum_{n \in S} k_{n} \longrightarrow 0
$$

and the associated long cohomology sequence

$$
0 \rightarrow H^{0}(X, L) \stackrel{\alpha}{\longrightarrow} H^{0}(Y, M) \stackrel{\beta}{\longrightarrow} k^{\delta} \rightarrow H^{1}(X, L) \rightarrow H^{1}(Y, M) \rightarrow 0
$$

from which we immediately get the upper bound on $h^{0}(X, L)$ stated in (13).

Fix $M \in \operatorname{Pic} Y$, recall the description of the fiber of $\nu^{*}$ over $M$ given in 1.1.4. Thus every $L \in F_{M}(X)$ is of the form $L=L^{(\underline{c})}$ for some $\underline{c} \in\left(k^{*}\right)^{\delta-\gamma+1}$. For convenience, we use the same set-up of 1.1.4, in particular we set $c_{j}=1$ for $\delta-\gamma \leq j \leq \delta$. 
To compute $H^{0}(X, L)$, set $l:=h^{0}(Y, M)$ and pick a basis $s_{1}, \ldots, s_{l}$ for $H^{0}(Y, M)$. Let $s \in H^{0}(Y, M)$, so $s=\sum_{1}^{l} x_{i} s_{i}$ where $x_{i} \in k$. Now $s$ descends to a section of $L$ (i.e. $s$ lies in the image of $\alpha$ in (17)) if and only if

$$
\sum_{i=1}^{l} x_{i}\left(s_{i}\left(q_{2}^{j}\right)-c_{j} s_{i}\left(q_{1}^{j}\right)\right)=0 \quad \forall j=1, \ldots, \delta .
$$

The above is a linear system of $\delta$ homogeneous equations in the $l$ unknowns $x_{1}, \ldots, x_{l}$. The space of its solutions, $\Lambda(\underline{c})$, is identified with $H^{0}\left(X, L^{(\underline{c})}\right)$. Now, $\Lambda(\underline{c})$ is a linear subspace of $H^{0}(Y, M)$ of dimension at least $l-\delta$. Hence $h^{0}(X, L)=\operatorname{dim} \Lambda(\underline{c}) \geq l-\delta$, proving (13).

Part (iii). Assume $l=h^{0}(Y, M) \geq \delta$; denote by $A(\underline{c})$ the $\delta \times l$ matrix of the system (18). By what we said

$$
h^{0}\left(X, L^{(\underline{c})}\right)=\operatorname{dim} \Lambda(\underline{c})=l-\operatorname{rank} A(\underline{c})
$$

and

$$
W_{M}(X) \cong\{\underline{c}: S(\underline{c}) \neq 0\}=\{\underline{c}: \operatorname{rank} A(\underline{c}) \lessgtr l\} .
$$

We shall prove that $A(\underline{c})$ has rank $\delta$ unless $\underline{c}$ lies in a proper closed subset of $\left(k^{*}\right)^{\delta}$. For that, we apply the assumption (14) to choose the basis for $H^{0}(Y, M)$ as follows. First, up to renaming each pair of branches we can assume that $h(j)=1$ for every $j$. By (14) we can pick $\delta$ linearly independent $s_{1}, \ldots s_{\delta} \in H^{0}(M)$ such that

$$
s_{i}\left(q_{1}^{j}\right)= \begin{cases}1 & \text { if } i=j \\ 0 & \text { if } j \neq i,(j=1, \ldots, \delta) .\end{cases}
$$

If $l>\delta$ we choose the remaining basis elements however we like. Set $b_{i}^{j}:=s_{i}\left(q_{2}^{j}\right) \in k$. Then the matrix $A(\underline{c})$ contains a $\delta \times \delta$ minor, $B(\underline{c})$, (the minor given by the first $\delta$ columns) whose diagonal is

$$
\left(c_{1}-b_{1}^{1}, \ldots, c_{\delta}-b_{\delta}^{\delta}\right)
$$

and such that the $c_{j}$ do not appear anywhere else in $B(\underline{c})$. Therefore the determinant of $B(\underline{c})$ is a nonzero polynomial in the $c_{j}$. This proves that the locus where the matrix has maximal rank (equal to $\delta$ ) is open, non empty.

Suppose $\delta=l$, then $B(\underline{c})=A(\underline{c})$. By (20) $W_{M}(X)$ is naturally identified with the locus of points of $F_{M}(X)$ where $\operatorname{det} A(\underline{c})$ vanishes. We conclude that $W_{M}(X)$ has pure dimension $\operatorname{dim} W_{M}(X)=\delta-\gamma$ proving (iii).

Moreover, looking at the matrix $A(\underline{c})$ it is easy to check that for a general $L^{(\underline{c})} \in W_{M}(X)$ the rank of $A(\underline{c})$ is equal to $\delta-1$; hence by (19) we get $h^{0}(X, L)=1$ proving (15).

If $l>\delta$, then $W_{M}(X)=F_{M}(X)$ by part (13). Furthermore, by (19)

$$
h^{0}\left(X, L^{(\underline{c})}\right)=l-\operatorname{rank} A(\underline{c}) \geq l-\delta .
$$

By looking at the matrix $A(\underline{c})$, we see that $h^{0}\left(X, L^{(\underline{c})}\right)=l-\delta$ holds on the non empty open subset where $\operatorname{det} B(\underline{c})$ does not vanish; this proves (15).

Lemma 2.2. Let $\nu: Y \rightarrow X$ be the normalization of $X$ and let $\underline{d} \in \Sigma^{s s}(X)$. For a general $M \in \operatorname{Pic}^{\underline{d}} Y$ we have

(i) $h^{0}(Y, M)=\delta$;

(ii) $M$ satisfies condition (14) w.r.t. a suitable $h:\{1, \ldots, \delta\} \rightarrow\{1,2\}$. 
(iii) $\operatorname{dim} W_{M}(X)=\delta-\gamma$;

(iv) The general $L$ in $W_{M}(X)$ satisfies $h^{0}(X, L)=1$.

Proof. Using the notation of 1.1.1, $Y=\coprod C_{i}$ with $C_{i}$ smooth of genus $g_{i}$, and $X=\cup \overline{C_{i}}$. The fact that $\underline{d}$ is semistable implies that $d_{i} \geq p_{a}\left(\overline{C_{i}}\right)-1 \geq g_{i}-1$ for every $i=1, \ldots, \gamma$. Therefore for $M$ general in $\operatorname{Pic} \underline{d} Y$

$$
h^{0}(Y, M)=\sum_{i}\left(d_{i}-g_{i}+1\right)=g-1-\sum_{i} g_{i}+\gamma=\delta .
$$

Let us prove (ii). We use definition 1.3.2 (A) of a semistable multidegree; $\Gamma_{X}$ of $X$ can be oriented so that, if $b_{i}$ denotes the number of edges pointing at $C_{i}$, then for all $i=1, \ldots, \gamma$

$$
d_{i}=g_{i}-1+b_{i}
$$

Any such orientation gives us a choice of branches over each node. Namely, for every $n_{j} \in X_{\text {sing }}$ we denote $q_{2}^{j}$ the branch corresponding to the ending half-edge of $n_{j}$. We claim that (14) holds with respect to the map $h(j)=2$ for every $j$. Indeed

$$
h^{0}\left(Y, M\left(-\sum_{j=1}^{\delta} q_{2}^{j}\right)\right)=\sum_{i=1}^{\gamma} h^{0}\left(C_{i}, M\left(-\sum_{j=1}^{\delta} q_{2}^{j}\right)_{\mid C_{i}}\right) .
$$

Now by (21)

$$
\operatorname{deg}_{C_{i}} M\left(-\sum_{j=1}^{\delta} q_{2}^{j}\right)=d_{i}-b_{i}=g_{i}-1
$$

hence ( $M$ being general) $h^{0}\left(C_{i}, M\left(-\sum_{j=1}^{\delta} q_{2}^{j}\right)_{\mid C_{i}}\right)=0$ for every $i$. We conclude that, by part (ii),

$$
h^{0}\left(Y, M\left(-\sum_{j=1}^{\delta} q_{2}^{j}\right)\right)=0=h^{0}(Y, M)-\delta
$$

so that (14) is satisfied.

Now, applying 2.1(ii), we get $\operatorname{dim} W_{M}(X)=\delta-\gamma$ and $h^{0}(X, L)=1$ for a general $L \in W_{M}(X)$. So (iii) and (iv) are proved.

Corollary 2.3. Let $\underline{d} \in \Sigma^{s s}(X)$ and let $L$ be a general line bundle in $\operatorname{Pic} \underline{d} X$. For every subcurve $Z \subseteq X$ we have $h^{0}\left(Z, L_{Z}\right)=d_{Z}-g_{Z}+1$.

Proof. It suffices to assume $Z$ connected (by (3) $)$. Consider the normalization $\nu: Y=\cup C_{i} \rightarrow X$ of $X$ and $\nu^{*} L=M=\left(L_{1}, \ldots, L_{\gamma}\right)$ with $L_{i} \in \operatorname{Pic}^{d_{i}} C_{i}$. Then $L_{i}$ is general in $\operatorname{Pic}^{d_{i}} C_{i}$ (as $L$ is general in Pic $\left.\underline{d} X\right)$; since $d_{i} \geq g_{i}-1$ (as $\underline{d}$ is semistable) we get that $h^{0}\left(C_{i}, L_{i}\right)=d_{i}-g_{i}+1$.

Now, denote $Z^{\nu} \rightarrow Z$ the normalization of $Z$, order the irreducible components of $X$ so that the first $\gamma_{Z}$ are the irreducible components of $Z$, set $S=Z_{\text {sing }}$, so that $g_{Z}=\sum_{i=1}^{\gamma_{Z}} g_{i}+\delta_{S}-\gamma_{Z}+1$. Let $M_{Z^{\nu}}$ be the restriction of $M$ to $Z^{\nu}$, then

$$
h^{0}\left(Z^{\nu}, M_{Z^{\nu}}\right)=\sum_{i=1}^{\gamma_{Z}} h^{0}\left(C_{i}, L_{i}\right)=\sum_{i=1}^{\gamma_{Z}}\left(d_{i}-g_{i}+1\right)=d_{Z}-g_{Z}+\delta_{S}+1 .
$$


Now, since $\underline{d}$ is semistable, $d_{Z} \geq g_{Z}-1$ hence $h^{0}\left(Z^{\nu}, M_{Z^{\nu}}\right) \geq \delta_{S}$. Moreover, recall that by 2.2 (iii) $M$ satisfies condition (14); it is straightforward to check that the analogue holds for $M_{Z^{\nu}}$, i.e. for a suitable choice of branches,

$$
h^{0}\left(Z^{\nu}, M_{Z^{\nu}}\left(-\sum_{j=1}^{\delta_{S}} q_{h(j)}^{j}\right)\right)=h^{0}\left(M_{Z^{\nu}}\right)-\delta_{S}=0
$$

This enables us to apply 2.1(15) to $Z^{\nu} \rightarrow Z$, thus getting

$$
h^{0}\left(Z, L_{Z}\right)=h^{0}\left(Z^{\nu}, M_{Z^{\nu}}\right)-\delta_{S}=d_{Z}-g_{Z}+\delta_{S}+1-\delta_{S}=d_{Z}-g_{Z}+1 .
$$

2.2. Basic cases. Recall the notation of 1.1.1, in particular (4). The following simple fact will be used various times.

Remark 2.4. Let $\nu_{S}: Y_{S} \rightarrow X$ be the normalization of $X$ at one node (i.e. $S=\{n\})$. Let $M \in \operatorname{Pic} Y_{S}$ be such that $h^{0}(M) \geq 2$. If $h^{0}\left(M\left(-q_{1}-q_{2}\right)\right)=$ $h^{0}(M)-2$, every $L \in F_{M}(X)$ satisfies $h^{0}(X, L)=h^{0}\left(Y_{S}, M\right)-1$

To prove it, pick $L \in F_{M}(X)$ and consider the cohomology sequence

$$
0 \rightarrow H^{0}(X, L) \stackrel{\alpha}{\longrightarrow} H^{0}\left(Y_{S}, M\right) \stackrel{\beta}{\longrightarrow} k \rightarrow H^{1}(X, L) \rightarrow H^{1}\left(Y_{S}, M\right) \rightarrow 0
$$

(associated to (16)). It suffices to show that $\beta$ is non zero. The assumption $h^{0}\left(M\left(-q_{1}-q_{2}\right)\right)=h^{0}(M)-2$ implies that $h^{0}\left(M\left(-q_{h}\right)\right)=h^{0}(M)-1$ for $h=1,2$; hence $M$ has a section $s$ vanishing at $q_{1}$ but not at $q_{2}$; but then $\beta(s) \neq 0$.

2.2.1. Let $S \subset X_{\text {sing }}$ and consider the partial normalization $Y_{S} \rightarrow X$. Fix a finite set $S^{\prime}$ of points of $X$ (usually $S^{\prime} \subseteq S$ ). For any $M \in \operatorname{Pic} Y_{S}$ set

$$
W_{M}\left(X, S^{\prime}\right):=\left\{L \in W_{M}(X): \forall s \in H^{0}(X, L) \exists n \in S^{\prime}: s(n)=0\right\}
$$

or equivalently (since $S^{\prime}$ is finite)

$$
W_{M}\left(X, S^{\prime}\right):=\left\{L \in W_{M}(X): \exists n \in S^{\prime}: s(n)=0 \quad \forall s \in H^{0}(X, L)\right\} .
$$

If $S=X_{\text {sing }}$ then $W_{M}(X, S)$ is equal to the set of points in $W_{M}(X)$ which do not lie in $\alpha \frac{d}{X}\left(\dot{X}^{\underline{d}}\right)$, where $\underline{d}=\underline{\operatorname{deg}} M$.

Lemma 2.5. Fix $\nu_{S}: Y_{S} \rightarrow X$ and let $M \in \operatorname{Pic}^{d} Y_{S}$ be such that

$$
h^{0}\left(Y_{S}, M\right)=1 .
$$

(1) If there exists $n_{j} \in S$ such that $h^{0}\left(Y_{S}, M\left(-q_{1}^{j}\right)\right) \neq h^{0}\left(Y_{S}, M\left(-q_{2}^{j}\right)\right)$, then $W_{M}(X)=\emptyset$.

(2) If $h^{0}\left(Y_{S}, M\left(-q_{1}^{j}\right)\right)=h^{0}\left(Y_{S}, M\left(-q_{2}^{j}\right)\right)$ for every $j$, there are two cases.

(a) If $h^{0}\left(Y_{S}, M\left(-q_{h}^{j}\right)\right)=0$ for every $j$ and $h$, then $Y_{S}$ is connected and there exists a $L_{M} \in F_{M}(X)$ such that $W_{M}(X)=\left\{L_{M}\right\}$ and $h^{0}\left(L_{M}\right)=1$. Moreover $W_{M}(X, S)=\emptyset$ (hence $L_{M} \in \alpha_{X}^{d}\left(\dot{X}^{d}\right)$ ).

(b) If there exists $j$ for which $h^{0}\left(Y_{S}, M\left(-q_{1}^{j}\right)\right)=h^{0}\left(Y_{S}, M\left(-q_{2}^{j}\right)\right)=1$, then $W_{M}(X, S)=W_{M}(X)$. Moreover: if $h^{0}\left(Y_{S}, M\left(-q_{h}^{j}\right)\right)=1$ for every $j$ then $W_{M}(X)=F_{M}(X)$; otherwise $W_{M}(X)=\left\{L_{M}\right\}$. 
Proof. Let $s \in H^{0}(M)$ be a nonzero section. In case (11) we are assuming that (up to switching the branches over $n_{j}$ ) $s\left(q_{1}^{j}\right)=0$ while $s\left(q_{2}^{j}\right) \neq 0$, so obviously $s$ does not descend to a section of any $L \in F_{M}(X)$.

For case (2a) suppose, by contradiction, that $Y_{S}=\coprod_{1}^{\gamma} Y_{i}$ is not connected. Then $h^{0}(Y, M)=\oplus h^{0}\left(Z_{i}, M_{Z_{i}}\right)=1$ so that there is only one component, say $Z_{1}$ such that $h^{0}\left(Z_{1}, M_{Z_{1}}\right) \neq 0$. Pick $q=q_{h}^{j} \in Z_{2}$, then $\left(\right.$ as $h^{0}\left(Z_{2}, M_{Z_{2}}\right)=0$ ) every $s \in H^{0}(M)$ vanishes at $q$ so that $h^{0}(M(-q))=h^{0}(M)=1$ contradicting the hypothesis. So $Y$ is connected. Now any nonzero $s \in H^{0}(Y, M)$ satisfies $s\left(q_{h}^{j}\right) \neq 0$ for $j=1, \ldots, \delta$ and $h=1,2$. Let $c_{j}:=s\left(q_{2}^{j}\right) / s\left(q_{1}^{j}\right) \in k^{*}$ and $\underline{c}=\left(c_{1}, \ldots, c_{\delta}\right)$; then $\underline{c}$ does not depend on the choice of $s$, as $h^{0}(M)=1$. Using the construction of 1.1 .4 set $L_{M}=L^{(\underline{c})}$; we get $W_{M}(X)=\left\{L_{M}\right\}$ and obviously $s$ descends to a section of $L_{M}$ that does not vanish at any $n_{j}$. So, $W_{M}(X, S)$ is empty, and by construction, $h^{0}\left(X, L_{M}\right)=1$.

In case (2b), it is clear that for every $L \in W_{M}(X)$ and $s \in H^{0}(L)$ we have $s\left(n_{j}\right)=0$, hence $W_{M}(X, S)=W_{M}(X)$. The last sentence is proved similarly.

Lemma 2.6. Let $\nu_{S}: Y_{S} \rightarrow X$ be the normalization of $X$ at one node (i.e. $S=\{n\})$. Let $M \in \operatorname{Pic}^{d} Y_{S}$ be such that $h^{0}\left(Y_{S}, M\right) \geq 2$.

Then $W_{M}(X)=F_{M}(X)$ and the following cases occur.

(1) If $h^{0}\left(M\left(-q_{1}-q_{2}\right)\right)=h^{0}(M)-2$ then $W_{M}(X, S)=\emptyset$ and $h^{0}(L)=$ $h^{0}(M)-1$ for every $L \in F_{M}(X)$.

(2) If $h^{0}\left(M\left(-q_{1}-q_{2}\right)\right)=h^{0}\left(M\left(-q_{h}\right)\right)=h^{0}(M)-1$ for $h=1$, 2 then $Y_{S}$ is connected and $W_{M}(X, S)=W_{M}(X) \backslash\left\{L_{M}\right\}$ for a uniquely determined $L_{M} \in W_{M}(X)$ (hence $L_{M} \in \alpha_{X}^{d}\left(\dot{X}^{d}\right)$ ). Moreover $h^{0}\left(L_{M}\right)=h^{0}(M)$ while for every $L \in W_{M}(X)-\left\{L_{M}\right\}$ we have $h^{0}(L)=h^{0}(M)-1$.

(3) If $h^{0}\left(M\left(-q_{1}\right)\right)=h^{0}(M)-1$ and $h^{0}\left(M\left(-q_{2}\right)\right)=h^{0}(M)$ then $F_{M}(X)=$ $W_{M}(X, S)$. Moreover $h^{0}(L)=h^{0}(M)-1$ for every $L \in F_{M}(X)$.

(4) If $h^{0}\left(M\left(-q_{1}\right)\right)=h^{0}\left(M\left(-q_{2}\right)\right)=h^{0}(M)$ then $F_{M}(X)=W_{M}(X, S)$. Moreover $h^{0}(L)=h^{0}(M)$ for every $L \in F_{M}(X)$.

Proof. Pick $L \in F_{M}(X)$ and consider the cohomology sequence (22). It yields that $\alpha\left(H^{0}(X, L)\right)$ has codimension at most 1, i.e. that $h^{0}(L) \geq$ $h^{0}(Y, M)-1 \geq 1$ so that $W_{M}(X)=F_{M}(X)$.

In the sequel shall omit the subscript $S$.

In case (11), $H^{0}\left(Y, M\left(-q_{1}-q_{2}\right)\right)$ has codimension 2 hence $\alpha\left(H^{0}(X, L)\right)$ cannot be contained in it. Therefore $H^{0}(X, L)$ contains sections that do not vanish at $n$. The rest has been proved in remark 2.4.

For the remaining cases, note that every section of $H^{0}\left(M\left(-q_{1}-q_{2}\right)\right)$ descends to a section of every $L \in F_{M}(X)$.

Case (2). To show that $Y$ is connected, suppose by contradiction that $Y=Y_{1} \amalg Y_{2}$, then (say) $q_{1} \in Y_{1}$ and $q_{2} \in Y_{2}$ and $h^{0}(M)=h^{0}\left(Y_{1}, M_{1}\right)+$ $h^{0}\left(Y_{2}, M_{2}\right)$ (denoting $\left.M_{i}=M_{Y_{i}}\right)$. Furthermore

$h^{0}\left(M_{1}\right)+h^{0}\left(M_{2}\right)-1=h^{0}(M)-1=h^{0}\left(M\left(-q_{1}\right)\right)=h^{0}\left(M_{1}\left(-q_{1}\right)\right)+h^{0}\left(M_{2}\right)$

hence $h^{0}\left(M_{1}\left(-q_{1}\right)\right)=h^{0}\left(M_{1}\right)-1$. Similarly, $h^{0}\left(M_{2}\left(-q_{2}\right)\right)=h^{0}\left(M_{2}\right)-1$. But then $h^{0}\left(M\left(-q_{1}-q_{2}\right)\right)=h^{0}\left(M_{1}\left(-q_{1}\right)\right)+h^{0}\left(M_{2}\left(-q_{2}\right)\right)=h^{0}(M)-2$ which is a contradiction. 
Now, there exists $s \in H^{0}(M)$ such that $s\left(q_{h}\right) \neq 0$ for $h=1,2$. Thus

$$
H^{0}(M)=H^{0}\left(M\left(-q_{1}-q_{2}\right)\right) \oplus k s
$$

Set $c=\frac{s\left(q_{2}\right)}{s\left(q_{1}\right)}$ and let $L_{M}=L^{(c)}$ (as in 1.1.4). The $s$ descends to a section $\bar{s} \in H^{0}\left(L_{M}\right)$ such that $\bar{s}(n) \neq 0$. Hence $L_{M} \notin W_{M}(X, S)$ and $h^{0}\left(L_{M}\right)=$ $h^{0}(M)$. Now, $L_{M}$ is uniquely determined, indeed if $s^{\prime} \in H^{0}(M)$ is another section such that $s^{\prime}\left(q_{h}\right) \neq 0$ for $h=1,2$, then by (25) $s^{\prime}=a t+b s$ for $t \in H^{0}\left(M\left(-q_{1}-q_{2}\right)\right)$ and $a, b \in k$ with $b \neq 0$. Thus $c=\frac{s^{\prime}\left(q_{2}\right)}{s^{\prime}\left(q_{1}\right)}$. This proves that for every $L \in W_{M}(X)$ such that $L \notin W_{M}(X, S)$ we have $L=L_{M}$.

In case (3), $H^{0}\left(M\left(-q_{1}-q_{2}\right)=H^{0}\left(M\left(-q_{1}\right)\right)\right.$ and these are the only sections that can be pull backs of sections of any $L \in F_{M}(X)$. Case (4) is obvious.

2.3. Divisors imposing independent conditions. Let $Y_{S} \rightarrow X$ be some partial normalization of $X$ and let $M \in \operatorname{Pic} Y_{S}$. The goal of this subsection is to bound the dimension of the locus of $L \in W_{M}(X)$ which are not contained in the image of the Abel map (i.e. with the notation of 2.2.1 the dimension of $\left.W_{M}(X, S)\right)$. The easy cases, $h^{0}\left(Y_{S}, M\right)=1$ or $\# S=1$, are dealt with by Lemmas 2.5 and 2.6. To treat the general case we introduce the following.

Definition 2.7. Let $Y$ be a nodal curve (possibly not connected). Let $M \in \operatorname{Pic} Y$ and let $E$ be a Cartier divisor on $Y$.

(A) We say that $E$ is admissibile for $M$ if for every subcurve $V \subseteq Y$ we have $0 \leq \operatorname{deg}_{V} E \leq h^{0}\left(V, M_{V}\right)$.

(B) We say that $E$ imposes independent conditions on $M$ if $E$ is admissible for $M$ and if $h^{0}\left(V, M(-E)_{V}\right)=h^{0}\left(V, M_{V}\right)-\operatorname{deg}_{V} E$ for every subcurve $V \subseteq Y$.

(C) Let $R \subset Y \backslash Y_{\text {sing }}$, we denote by $\mathcal{A}(M, R)$ the set of all admissible divisors for $M$ with support contained in $R$.

Remark 2.8. If $R$ in part ( $(\mathrm{C})$ is finite, then the set $\mathcal{A}(M, R)$ is also finite.

Let $C$ be a smooth irreducible curve, Definition 2.7 gives back the classical one. Fix a finite subsetset $R \subset C$; then every admissible divisor $E$ such that Supp $E \subset R$ imposes independent conditions on the general $L \in \operatorname{Pic}^{d} C$. More generally

Lemma 2.9. Let $\nu: Y \rightarrow X$ be the normalization of $X$ and $R \subset Y$ a finite subset. Let $\underline{d} \in \Sigma^{s s}(X)$ and $M \in \operatorname{Pic} \underline{\underline{d}} Y$ a general point. Then every divisor $E \in \mathcal{A}(M, R)$ imposes independent conditions on $M$.

Proof. By Remark 2.8, it suffices to prove that a fixed $E$ imposes independent conditions on the general $M \in \operatorname{Pic}^{\underline{d}} Y$.

Set as usual $Y=\coprod_{i=1}^{\gamma} C_{i}$. Given $M$ and $E$ as in the statement, denote $M_{i}:=M_{C_{i}}, E_{i}:=E_{C_{i}}$ and $e_{i}=\operatorname{deg}_{C_{i}} E$. Now, for any line bundle $N$ on $Y$ and any subcurve $V \subset Y$ we have $H^{0}(V, N)=\oplus_{C_{i} \subset V} H^{0}\left(C_{i}, N_{C_{i}}\right)$. Therefore it suffices to prove that for every $i=1 \ldots, \gamma$

$$
h^{0}\left(C_{i}, M(-E)_{C_{i}}\right)=h^{0}\left(C_{i}, M_{i}\right)-e_{i} .
$$

Since $M$ is general in $\operatorname{Pic}^{\underline{d}} Y=\prod \operatorname{Pic}^{d_{i}} C_{i}$, every $M_{i}$ is general in $\operatorname{Pic}^{d_{i}} C_{i}$. The fact that $\underline{d}$ is semistable implies that $d_{i} \geq p_{a}\left(C_{i}\right)-1 \geq g_{i}-1\left(g_{i}\right.$ is 
the genus of $\left.C_{i}\right)$ hence $h^{0}\left(C_{i}, M_{i}\right)=d_{i}-g_{i}+1$. Now by (A) of 2.7 we have $e_{i} \leq d_{i}-g_{i}+1$ hence

$$
\operatorname{deg}_{C_{i}} M(-E)=d_{i}-e_{i} \geq g_{i}-1 .
$$

At this point, observe that $M_{i}\left(-E_{i}\right)$ is a general point in $\operatorname{Pic}^{d_{i}-e_{i}} C_{i}\left(E_{i}\right.$ is fixed and $M_{i}$ is general in $\mathrm{Pic}^{d_{i}} C_{i}$ ) and hence (by (26) )

$$
h^{0}\left(C_{i}, M_{i}\left(-E_{i}\right)\right)=d_{i}-e_{i}-g_{i}+1=h^{0}\left(C_{i}, M_{i}\right)-e_{i}
$$

as claimed.

Example 2.10. Let $\nu: Y \rightarrow X$ the normalization of $X, \underline{d} \in \Sigma^{s s}(X)$ and $M \in \operatorname{Pic}^{\underline{d}} Y$. Then there exists a choice of branches $h:\{1, \ldots, \delta\} \rightarrow\{1,2\}$ such that the divisor $\sum_{j=1}^{\delta} q_{h(j)}^{j}$ is admissible for $M$. In fact, the construction of such an admissible divisor $E$ has appeared in the proof of 2.2 ,

For the next result we need some notation. Recall that $\nu_{S}: Y_{S} \rightarrow X$ denotes the normalization of $X$ at $S$. Let $Z \subset X$ be a subcurve, we denote $Z_{S}:=\nu_{S}^{-1}(Z)$ the corresponding subcurve in $Y_{S}$, so that $Z_{S}$ is the normalization of $Z$ at $S \cap Z_{\text {sing }}$. Obviously every subcurve of $Y_{S}$ is of the form $Z_{S}$ for a unique $Z \subset X$. We shall often simplify the notation by setting $H^{0}\left(Z_{S}, M\right):=H^{0}\left(Z_{S}, M_{Z_{S}}\right)$ whenever no confusion is possible.

Proposition 2.11. Fix $\nu_{S}: Y_{S} \rightarrow X$ as above. Let $M \in \operatorname{Pic} Y_{S}$ be such that $h^{0}\left(Y_{S}, M\right) \geq \delta_{S}$ and for every $Z_{S} \subsetneq Y_{S}$

$$
h^{0}\left(Z_{S}, M_{Z_{S}}\right) \geq 1+\#\left(S \cap Z_{\text {sing }}\right) .
$$

If every $E \in \mathcal{A}\left(M, \nu_{S}^{-1}(S)\right)$ imposes independent conditions on $M$, then

$$
\operatorname{dim} W_{M}(X, S) \leq \begin{cases}\delta_{S}-\gamma_{S}-1 & \text { if } h^{0}(M)=\delta_{S} \\ \delta_{S}-\gamma_{S} & \text { if } h^{0}(M) \geq \delta_{S}+1\end{cases}
$$

Proof. We shall denote $l:=h^{0}\left(Y_{S}, M\right)$. By hypothesis, for every $q \in \nu^{-1}(S)$

$$
h^{0}\left(Y_{S}, M(-q)\right)=l-1
$$

indeed by (27) every such $q$ is admissible for $M$. Let $n \in S$ and set $\nu^{-1}(n)=$ $\left\{q_{1}, q_{2}\right\}$. Suppose $l=1$; then $\delta_{S}=1$. By (28) applied to $q_{1}$ and $q_{2}$, we are in case (2a) of Lemma 2.5. Hence $W_{M}(X, S)=\emptyset$ and we are done.

From now on, we assume $l \geq 2$.

Let $E=q_{1}+q_{2}$, then $E$ is admissible, i.e. $\operatorname{deg}_{Z_{S}} E \leq h^{0}\left(Z_{S}, M_{Z_{S}}\right)$, for every subcurve $Z_{S} \subset Y_{S}$. Indeed, for every $Z_{S}$, we have $h^{0}\left(Z_{S}, M_{Z_{S}}\right) \geq 1$ by (27). On the other hand $\operatorname{deg}_{Z_{S}} E \leq 2$ and equality holds iff $Z_{S}$ contains both $q_{1}$ and $q_{2}$, i.e. if and only if $Z$ is singular at $n$. In this case, $h^{0}\left(Z_{S}, M_{Z_{S}}\right) \geq 2$ by (27). Therefore, by hypothesis, for every $Z_{S}$

$$
h^{0}\left(Z_{S}, M\left(-q_{1}-q_{2}\right)\right)=h^{0}\left(Z_{S}, M_{Z_{S}}\right)-2 .
$$

Assume $\delta_{S}=1$. By (29) we are in case (11) of lemma 2.6. Thus $W_{M}(X, S)$ is empty and we are done. We continue by induction on $\delta_{S}$.

For every $j=1, \ldots, \delta$ set $S_{j}:=S \backslash\left\{n_{j}\right\}$. For any $\left\{j_{1}, j_{2}\right\} \subset\left\{1, \ldots, \delta_{S}\right\}$

$$
W_{M}(X, S)=\bigcup_{j=1}^{\delta} W_{M}\left(X, S_{j}\right)=W_{M}\left(X, S_{j_{1}}\right) \cup W_{M}\left(X, S_{j_{2}}\right)
$$


therefore it suffices to bound the dimension of $W_{M}\left(X, S_{j}\right)$ for a chosen pair of values of $j=1, \ldots, \delta$. We pick one of them and simplify the notation by setting $n=n_{j}$ and $T=S_{j}=S \backslash\{n\}$. We factor $\nu_{S}$

$$
\nu_{S}: Y_{S} \stackrel{\nu_{1}}{\longrightarrow} Y_{T} \stackrel{\nu_{T}}{\longrightarrow} X
$$

where $\nu_{T}$ is the normalization of $X$ at $T$ and $\nu_{1}$ the normalization at the remaining node $n$. We abuse notation by using the same names for points in $Y_{S}, Y_{T}$ and $X$ whenever the maps are local isomorphims (e.g. $n$ denotes a node in $Y_{T}$ and in $X$ ). The following is the basic diagram to keep in mind

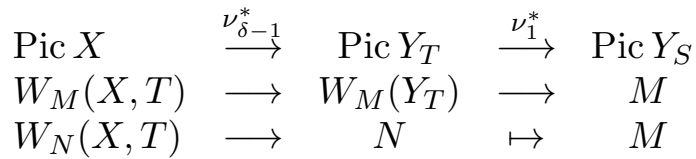

where $N \in F_{M}\left(Y_{T}\right)$; since $l \geq 2, F_{M}\left(Y_{T}\right)=\mathrm{W}_{M}\left(Y_{T}\right)$. By (29) and 2.4,

$$
h^{0}\left(Y_{T}, N\right)=l-1 \text {. }
$$

Case 1. The node $n$ lies in two different irreducible components of $X$.

By Lemma 2.12 part (ii) (applied with $R=\nu_{S}^{-1}(S \backslash n)$ ) every admissible divisor $E_{T}$ on $Y_{T}$ such that $\operatorname{Supp} E_{T} \subset \nu_{T}^{-1}(T)$ imposes independent conditions on $N$. Therefore we can use induction $(\# T=\# S-1)$ and obtain

$$
\operatorname{dim} W_{N}(X, T) \leq \begin{cases}\delta_{S}-1-\gamma_{T}-1 & \text { if } h^{0}\left(Y_{T}, N\right)=\delta_{S}-1 \\ \delta_{S}-1-\gamma_{T} & \text { if } h^{0}\left(Y_{T}, N\right) \geq \delta_{S}\end{cases}
$$

i.e. using (32)

$$
\operatorname{dim} W_{N}(X, T) \leq \begin{cases}\delta_{S}-\gamma_{T}-2 & \text { if } l-1=\delta_{S}-1 \\ \delta_{S}-\gamma_{T}-1 & \text { if } l-1 \geq \delta_{S}\end{cases}
$$

If $n$ is not a separating node for $X$, then $F_{M}\left(Y_{T}\right)=\mathrm{W}_{M}\left(Y_{T}\right) \cong k^{*}$ and $\gamma_{S}=$ $\gamma_{T}$. Therefore, from diagram (31), $\operatorname{dim} W_{M}(X, T) \leq \operatorname{dim} W_{N}(X, T)+1$. So, using that $\delta_{S}-\gamma_{T}-1=\delta_{S}-\gamma_{S}$, we are done.

If $n$ is separating, then $\gamma_{S}=\gamma_{T}+1$. On the other hand $\operatorname{dim} F_{M}\left(Y_{T}\right)=0$, hence $\operatorname{dim} W_{M}(X, T) \leq \operatorname{dim} W_{N}(X, T)$. Again, we are done.

Case 2. The node $n$ lies in only one irreducible component of $X$.

Let $n \in \bar{C}$ and let $C \subset Y_{S}$ the component of $Y_{S}$ containing both $q_{1}$ and $q_{2}$. We are in the situation of Lemma 2.12 part (iii). We get that there exists a finite set $P \subset F_{M}\left(Y_{T}\right)$ such that for every $N \in \operatorname{Pic} Y_{T} \backslash P$, every admissible $E$ supported on $\nu_{T}^{-1}(T)$ imposes independent conditions on $N$. We can use induction on every $N \in W_{M}\left(Y_{T}\right)$ such that $N \notin P$. We obtain

$$
\operatorname{dim} W_{N}(X, T) \leq \begin{cases}\delta_{S}-1-\gamma_{T}-1 & \text { if } h^{0}\left(Y_{T}, N\right)=\delta_{S}-1 \\ \delta_{S}-1-\gamma_{T} & \text { if } h^{0}\left(Y_{T}, N\right) \geq \delta_{S}\end{cases}
$$

Consider diagram (31) and note that now $\operatorname{dim} W_{M}\left(Y_{T}\right)=\operatorname{dim} F_{M}\left(Y_{T}\right)=$ 1. Hence, away from the fibers over $P$, the dimension of every irreducible component of $W_{M}(X, T)$ is at most

$$
\operatorname{dim} W_{M}\left(Y_{T}\right)+\operatorname{dim} W_{N}(X, T) \leq \begin{cases}1+\delta-\gamma-2 & \text { if } l=\delta \\ 1+\delta-\gamma-1 & \text { if } l \geq \delta+1\end{cases}
$$

(using (32)) as wanted. 
It remains to bound the dimension of the fibers over every $N \in P$. Now, set $n=n_{1}$ and $T=\left\{n_{2}, \ldots, n_{\delta_{S}}\right\}$.

If $l \geq \delta_{S}+1$, i.e. if $h^{0}\left(Y_{T}, N\right) \geq \delta_{T}+1$ then

$$
\operatorname{dim} W_{N}(X)=\operatorname{dim} F_{N}(X)=\delta_{T}-\gamma_{T}+1=\delta_{S}-\gamma_{S} .
$$

The fiber of $W_{M}(X, T) \rightarrow W_{M}\left(Y_{T}\right)$ over $N$ is obviously contained in $W_{N}(X)$, hence it has dimension at most $\delta_{S}-\gamma_{S}$ and we are done.

Assume $\delta_{S}=l$. If (up to switching the branches over each node)

$$
h^{0}\left(Y_{T}, N\left(-q_{1}^{2}-\ldots-q_{1}^{\delta_{S}}\right)\right)=0,
$$

then, by 2.1 (iii), $W_{N}(X)$ has pure dimension equal to $\delta_{T}-\gamma_{S}=\delta_{S}-\gamma_{S}-1$. Hence the dimension of the fiber of $W_{M}(X, T)$ over $N$ is at most $\delta_{S}-\gamma_{S}-1$ and we are done. Let us complete the proof by showing that (33) holds for some choice of branches. Suppose $h^{0}\left(Y_{T}, N\left(-q_{1}^{2}-\ldots-q_{1}^{\delta_{S}}\right)\right) \geq 1$; we want to prove that $h^{0}\left(Y_{T}, N\left(-q_{1}^{2}-\ldots-q_{1}^{\delta_{S}-1}-q_{2}^{\delta_{S}}\right)\right)=0$. We argue by contradiction: let $h^{0}\left(N\left(-q_{1}^{2}-\ldots-q_{1}^{\delta_{S}-1}-q_{2}^{\delta_{S}}\right)\right) \geq 1$. Then of course

$$
h^{0}\left(N\left(-\sum_{j=2}^{\delta_{S}} q_{1}^{j}-q_{2}^{\delta_{S}}\right)\right) \geq 1 .
$$

Now $\nu_{1}^{*} N\left(-\sum_{j=2}^{\delta_{S}} q_{1}^{j}-q_{2}^{\delta_{S}}\right)=M\left(-\sum_{j=2}^{\delta_{S}} q_{1}^{j}-q_{2}^{\delta_{S}}\right)$ and we claim that

$$
h^{0}\left(Y_{S}, M\left(-\sum_{j=2}^{\delta_{S}} q_{1}^{j}-q_{2}^{\delta_{S}}\right)\right)=0,
$$

in contradiction with (34). Our claim holds because $E=\sum_{j=2}^{\delta_{S}} q_{1}^{j}+q_{2}^{\delta_{S}}$ is admissible for $M$. Indeed: $\operatorname{deg}_{Z_{S}} E \leq 1+\# T \cap Z_{\text {sing }}$ for every $Z_{S} \subset Y_{S}$. Hence, using assumption (27)

$$
\operatorname{deg}_{Z_{S}} E \leq 1+\# T \cap Z_{\text {sing }} \leq 1+\# S \cap Z_{\text {sing }} \leq h^{0}\left(Z_{S}, M\right)
$$

so $E$ is admissible for $M$ and we are done.

In the proof of Proposition 2.11 we used the following

Lemma 2.12. Let $\nu_{1}: Y_{S} \longrightarrow Y_{T}$ be the partial normalization of $Y_{T}$ at a unique node n. Let $M \in \operatorname{Pic} Y_{S}$ be such that for every subcurve $Z_{S} \subset Y_{S}$

$$
h^{0}\left(Z_{S}, M\right) \begin{cases}\geq 2 & \text { if } \nu_{1}^{-1}(n) \subset Z_{S} \\ \geq 1 & \text { otherwise }\end{cases}
$$

Let $R$ be a finite set of smooth points of $Y_{S}$. Assume that every divisor in $\mathcal{A}\left(M, \nu_{1}^{-1}(n) \cup R\right)$ imposes independent conditions on $M$.

(i) If $n$ lies in two irreducible components of $Y_{T}$, for any $N \in F_{M}\left(Y_{T}\right)$, every divisor in $\mathcal{A}\left(N, \nu_{1}(R)\right)$ imposes independent conditions on $N$.

(ii) If $n$ lies in only one irreducible component of $Y_{T}$, there exists a finite subset $P \subset F_{M}\left(Y_{T}\right)$ such that for every $N \in F_{M}\left(Y_{T}\right) \backslash P$, every divisor in $\mathcal{A}\left(N, \nu_{1}(R)\right)$ imposes independent conditions on $N$.

Proof. Let $\nu^{-1}(n)=\left\{q_{1}, q_{2}\right\}$. Formula (29) holds (with the same proof). For every $Z_{S} \subset Y_{S}$, denote $Z_{T}:=\nu_{1}\left(Z_{S}\right)$. We have by (29) and 2.4

$$
\text { if }\left\{q_{1}, q_{2}\right\} \subset Z_{S} \Longrightarrow h^{0}\left(Z_{T}, N_{Z_{T}}\right)=h^{0}\left(Z_{S}, M_{Z_{S}}\right)-1
$$


and

$$
\text { if }\left\{q_{1}, q_{2}\right\} \not \subset Z_{S} \Longrightarrow h^{0}\left(Z_{T}, N_{Z_{T}}\right)=h^{0}\left(Z_{S}, M_{Z_{S}}\right)
$$

because in this case $Z_{S} \cong Z_{T}$ via $\nu_{1}$. Thus for any $N \in F_{M}\left(Y_{T}\right)$, the number $h^{0}\left(Z_{T}, N_{Z_{T}}\right)$ depends only on $M$, and not on the choice of $N$. Therefore the set $\mathcal{A}\left(N, \nu_{1}(R)\right)$ depends only on $M$.

Pick $E_{T} \in \mathcal{A}\left(N, \nu_{1}(R)\right)$. Denote $E_{S}:=\nu_{1}^{*}\left(E_{T}\right)$, and observe that $\nu_{1}$ is an isomorphism locally at every point in $\operatorname{Supp} E_{S}$. Hence

$$
\operatorname{deg}_{Z_{S}} E_{S}=\operatorname{deg}_{Z_{T}} E_{T} \leq h^{0}\left(Z_{T}, N\right) \leq h^{0}\left(Z_{S}, M\right) .
$$

Therefore $E_{S}$ imposes independent conditions on $M$, i.e.

$$
h^{0}\left(Z_{S}, M\left(-E_{S}\right)\right)=h^{0}\left(Z_{S}, M\right)-\operatorname{deg}_{Z_{S}} E_{S} .
$$

If $\left\{q_{1}, q_{2}\right\} \not \subset Z_{S}, \nu_{1}$ induces an isomorphism $Z_{S} \cong Z_{T}$, hence by (37) and (38) we get $h^{0}\left(Z_{T}, N\left(-E_{T}\right)\right)=h^{0}\left(Z_{S}, M\left(-E_{S}\right)\right)=h^{0}\left(Z_{T}, N\right)-\operatorname{deg}_{Z_{T}} E_{T}$ as wanted. So we need only consider the case $\left\{q_{1}, q_{2}\right\} \subset Z_{S}$.

For part (ii), let $q_{1} \in C_{1}$ and $q_{2} \in C_{2}$. Set $e_{i}:=\operatorname{deg}_{C_{i}} E$ and $l_{i}:=$ $h^{0}\left(C_{i}, M_{C_{i}}\right)=h^{0}\left(C_{i}, N_{C_{i}}\right)$. Consider the usual sequence

$$
0 \longrightarrow H^{0}\left(Z_{T}, N\left(-E_{T}\right)\right) \longrightarrow H^{0}\left(Z_{S}, M\left(-E_{S}\right)\right) \stackrel{\beta}{\longrightarrow} k \longrightarrow \ldots
$$

If $E_{T}$ is such that $e_{i} \leq l_{i}-1$ for $i=1,2$ then $E_{S}+q_{1}+q_{2}$ imposes independent conditions on $M$. We get $h^{0}\left(Z_{S}, M\left(-E_{S}-q_{1}-q_{2}\right)\right)=h^{0}\left(Z_{S}, M\left(-E_{S}\right)\right)-2$, hence $h^{0}\left(Z_{T}, N\left(-E_{T}\right)\right)=h^{0}\left(Z_{S}, M\left(-E_{S}\right)\right)-1$. By (37) and (38) we get

$$
h^{0}\left(Z_{T}, N\left(-E_{T}\right)\right)=h^{0}\left(Z_{S}, M\right)-\operatorname{deg}_{Z_{T}} E_{S}-1=h^{0}\left(Z_{T}, N\right)-\operatorname{deg}_{Z_{T}} E_{T}
$$

as wanted.

$E_{T}$ is admissible, hence $l_{i} \geq e_{i}$; so only two cases remain.

Case 1: $e_{1}=l_{1}$ and $e_{2}=l_{2}-1$. Then $H^{0}\left(C_{1}, M\left(-E_{S}\right)\right)=0, h^{0}\left(C_{2}, M\left(-E_{S}\right)\right)=$ 1 and $h^{0}\left(C_{2}, M\left(-E_{S}-q_{2}\right)\right)=0$. Then all sections in $H^{0}\left(Z_{S}, M\left(-E_{S}\right)\right)$ vanish at $q_{1}$ while there exist sections that do not vanish at $q_{2}$. Hence $\beta$ is surjective and we are done.

Case 2: $l_{i}=e_{i}$ for $i=1,2$. Let $Z_{T}:=\nu_{1}\left(C_{1} \cup C_{2}\right) \subset Y_{T}$. By (35)

$$
e_{1}+e_{2}=\operatorname{deg}_{Z_{T}} E_{T} \leq h^{0}\left(Z_{T}, N\right)=h^{0}\left(Z_{S}, M\right)-1 \leq l_{1}+l_{2}-1
$$

which is possible only if at least one $e_{i}$ is less than $l_{i}$. So Case 2 does not occur and (ii) is proved.

Now part (iii). Call $C \subset Y_{S}$ the component of $Y_{S}$ containing both $q_{1}$ and $q_{2}$, and $D:=\nu_{1}(C)$. Set $e_{D}=\operatorname{deg}_{D} E_{T}=\operatorname{deg}_{C} E_{S}$; and (by (35))

$$
l_{D}:=h^{0}(C, M)=h^{0}(D, N)+1
$$

so that $e_{D} \leq l_{D}-1$. If $e_{D} \leq l_{D}-2$ then $E_{S}+q_{1}+q_{2}$ is admissible for $M$. Hence for every $Z_{S} \subset Y_{S}$ we have $h^{0}\left(Z_{S}, M\left(-E_{S}-q_{1}-q_{2}\right)\right)=$ $h^{0}\left(Z_{S}, M(-E)\right)-2$ so that (using remark 2.4)

$$
h^{0}\left(Z_{T}, N\left(-E_{T}\right)\right)=h^{0}\left(Z_{S}, M\left(-E_{S}\right)\right)-1=h^{0}\left(Z_{T}, N\right)-\operatorname{deg}_{Z_{T}} E_{T} .
$$

We are left with case is $e_{D}=l_{D}-1$. Then $h^{0}\left(C, M\left(-E_{S}\right)\right)=1$ and part (2a) of Lemma 2.5 applies. We obtain that there exists a unique line bundle in Pic $D$ which pulls back to $M\left(-E_{S}\right)_{C}$ and having $h^{0}=1$. This in turn determines a (unique) line bundle $N_{D}$ on $D$ which pulls back to $M_{C}$, and finally a unique line bundle on $Y_{T}$ which pulls back to $M$ and restricts to 
$N_{D}$ on $D$. This last line bundle on $Y_{T}$ is uniquely determined by $E_{T}$, so we shall denote it by $N^{E_{T}}$. Set $P:=\left\{N \in F_{M}\left(Y_{T}\right): N=N^{E_{T}}\right.$ for some $\left.E_{T}\right\}$. We just showed that for any $N \in F_{M}\left(Y_{T}\right) \backslash P$, every $E_{T} \in \mathcal{A}\left(N, \nu_{1}(R)\right)$ imposes independent conditions on $N$. The finiteness of the set $P$ follows immediately from the finiteness of the set of $E_{T}$ 's.

Corollary 2.13. Let $Y \rightarrow X$ be the normalization of $X$ and $S=X_{\text {sing. If }}$ $\underline{d} \in \Sigma(X)$ and $M \in \operatorname{Pic}^{\underline{d}} Y$ is a general point then

$$
\operatorname{dim} W_{M}(X, S) \leq \delta-\gamma-1 .
$$

Proof. If $M$ is general, $h^{0}(Y, M)=\delta$ by 2.2. Moreover, as $\underline{d}$ is stable, (27) holds. Indeed for every $Z \subset X, Z^{\nu}=Z_{S}$ is the normalization of $Z$ and we have $d_{Z} \geq p_{a}(Z)=p_{a}\left(Z^{\nu}\right)+\# Z_{\text {sing }}$; hence $h^{0}\left(Z^{\nu}, M_{Z^{\nu}}\right) \geq \# Z_{\text {sing }}+1$. Finally, by Lemma 2.9, $M$ satisfies the assumption of Proposition 2.11.

\section{IRREDUCIBILITY AND DIMENSION}

3.1. Irreducible components of the Theta divisor. We are ready to prove that $W_{\underline{d}}(X)$ is irreducible for every stable multidegree $\underline{d}$. The stability assumption on $\underline{d}$ is essential, see examples 3.4 and 3.5 . If $|\underline{d}| \geq 1$ we shall use the Abel map $\alpha \frac{d}{X}$. If $|\underline{d}| \leq 0$, i.e. if $g=0,1$ the Abel map is not defined so we need to treat this case separately, which will be done in the following

Lemma 3.1. Let $X$ be a nodal connected curve of genus $g$ with $g=0,1$; let $\underline{d} \in \Sigma(X)$. Then

$$
W_{\underline{d}}(X)=\left\{\begin{array}{ll}
\emptyset & \text { if } \underline{d} \neq(0, \ldots, 0) \\
\left\{\mathcal{O}_{X}\right\} & \text { if } \underline{d}=(0, \ldots, 0)
\end{array} \quad \text { (hence } g=1\right)
$$

Proof. By hypothesis, $\forall \underline{d} \in \Sigma(X)$ we have $|\underline{d}|=-1,0$ depending on whether $g=0,1$. Recall that $X=\cup \overline{C_{i}}$ denotes the decomposition of $X$ in irreducible components. Let $L \in \operatorname{Pic} \underline{d} X$ and suppose that there exists a nonzero section $s \in H^{0}(X, L)$. Set

$$
Z^{-}:=\bigcup_{i: d_{i}<0} \overline{C_{i}} ; \quad Z^{0}:=\bigcup_{i: d_{i}=0} \overline{C_{i}} ; \quad Z^{+}:=\bigcup_{i: d_{i}>0} \overline{C_{i}} .
$$

Note that $Z^{-}=\emptyset \Leftrightarrow \underline{d}=(0, \ldots, 0)$. By contradiction, assume $Z^{-} \neq \emptyset$. Then $s$ vanishes along a non empty subcurve $Z \subset X$ which contains $Z^{-}$. Let $Z^{C}$ be the complementray curve of $Z$, so that $s$ does not vanish along any subcurve of $Z^{C}$. Since for every $n \in Z \cap Z^{C}$ we have $s(n)=0$, the degree of $s$ restricted to $Z^{C}$ satisfies

$$
d_{Z^{C}} \geq \# Z \cap Z^{C} \text {. }
$$

On the other hand, $g \leq 1$ implies $p_{a}\left(Z^{C}\right) \leq 1$ hence the stability of $\underline{d}$ yields

$$
d_{Z^{C}} \leq p_{a}\left(Z^{C}\right)+\# Z \cap Z^{C}<\# Z \cap Z^{C}
$$

(cf. 1.6) a contradiction with (42). Therefore $Z^{-}=\emptyset$; we obtain that, if $W_{\underline{d}}(X) \neq \emptyset$ then $\underline{d}=(0, \ldots, 0)$; in particular, $g=1$.

So let $\underline{d}=(0, \ldots, 0)$; of course $\mathcal{O}_{X} \in W_{\underline{d}}(X)$. Let $\nu: Y \rightarrow X$ be the normalization of $X$. We can factor $\nu$ as follows

$$
\nu: Y \longrightarrow Y_{Y} \stackrel{\nu_{T}}{\longrightarrow} X
$$


with $Y_{T}$ connected of compact type. Thus $\operatorname{Pic} Y \cong \operatorname{Pic} Y_{T}$ and $h^{0}\left(Y_{T}, \mathcal{O}_{Y_{T}}\right)=$ 1 because $Y_{T}$ is connected. Furthermore it is easy to see that $W_{(0, \ldots, 0)}\left(Y_{T}\right)=$ $\left\{\mathcal{O}_{Y_{T}}\right\}$ (using 2.5 and 2.6). To pass from $Y_{T}$ to $X$ we glue one pair of corresponding branches at the time; in each step we are in the situation of Lemma 2.5 (2a). Hence there exists a unique $L \in \mathrm{Pic}^{(0, \ldots, 0)} X$ such that $\nu_{T}^{*} L=\mathcal{O}_{Y_{T}}$, so $L=\mathcal{O}_{X}$ and we are done.

Recall that for $\underline{d}$ such that $\underline{d} \geq 0$ and $|\underline{d}| \geq 1$ we denote by $A_{\underline{d}}(X) \subset \operatorname{Pic} \underline{d} X$ the closure of the image of the Abel map $\alpha \frac{d}{X}$ (see 1.2.3). If $\underline{d} \in \Sigma(X)$ is such that $|\underline{d}|=-1,0$, we denote $A_{\underline{d}}(X):=W_{\underline{d}}(X)$.

Theorem 3.2. Let $X$ be a connected nodal curve of arithmetic genus g. Let $\underline{d}$ be a stable multidegree on $X$ such that $|\underline{d}|=g-1$. Then

(i) $W_{\underline{d}}(X)=A_{\underline{d}}(X)$, hence $W_{\underline{d}}(X)$ is irreducible of dimension $g-1$;

(ii) the general $\bar{L} \in W_{\underline{d}}(X)$ satisfies $h^{0}(X, L)=1$.

Proof. If $g=0,1$ the theorem follows from Lemma 3.1; so we assume $g \geq 2$. (ii) follows from (ii) and from 2.2 (iv).

Let $W$ be an irreducible component of $W_{\underline{d}}(X)$. By 1.10 we know that $\operatorname{dim} W=g-1$. We shall prove the Theorem by showing that if $A_{\underline{d}}(X)$ is not dense in $W$, i.e. if $W \neq \overline{W \cap \operatorname{Im} \alpha \frac{d}{X}}$, then $\operatorname{dim} W \leq g-2$, and hence $W$ must be empty.

Up to removing a proper closed subset of $W$, we can and will assume that

$$
W \cap \operatorname{Im} \alpha \frac{d}{X}=\emptyset .
$$

Consider $\nu: Y \rightarrow X$ the normalization of $X$, with $Y=\coprod_{1}^{\gamma} C_{i}$ and let $g_{i}$ be genus of $C_{i}$. Recall that $g=\sum_{1}^{\gamma} g_{i}+\delta-\gamma+1$.

We shall call $\rho$ the restriction to $W$ of the pull-back map $\nu^{*}$, so that

$$
\operatorname{Pic}^{\underline{d}} X \supset W \stackrel{\rho}{\longrightarrow} \rho(W) \subset \operatorname{Pic}^{\underline{d}} Y=\prod_{i=1}^{\gamma} \operatorname{Pic}^{d_{i}} C_{i}
$$

We shall bound the dimension of $W$ by analyzing $\rho$.

To say that $L \in \operatorname{Pic}^{\underline{d}} X$ does not lie in the image of $\alpha_{X}^{d}: \dot{X}^{d} \rightarrow \operatorname{Pic} X$ is to say that $L$ does not admit any section whose zero locus is contained in $\dot{X}$. In other words, setting $S=X_{\text {sing, }}$, we have $L \in W_{M}(X, S)$ (cf. 2.2.1). Therefore for every $M$ in $\rho(W)$ we have

$$
\rho^{-1}(M) \subset W_{M}(X, S) \subset W_{M}(X) .
$$

From now on we shall denote by $M$ a general point in $\rho(W)$. The proof is divided into four cases.

Case I. $\operatorname{dim} \rho(W) \leq \sum_{1}^{\gamma} g_{i}-2$.

It suffices to use that $\operatorname{dim} \rho^{-1}(M) \leq \operatorname{dim} F_{M}(X)=\delta-\gamma+1$. Then

$$
\operatorname{dim} W \leq \operatorname{dim} \rho(W)+\operatorname{dim} F_{M}(X) \leq \sum_{1}^{\gamma} g_{i}-2+\delta-\gamma+1=g-2 .
$$

Case II. $\operatorname{dim} \rho(W)=\sum_{1}^{\gamma} g_{i}$. 
Now $\rho$ is dominant, so that $M$ is general in $\operatorname{Pic} \underline{d} Y=\prod_{1}^{\gamma} \operatorname{Pic}^{d_{i}} C_{i}$. Then we can apply Corollary 2.13 which yields

$$
\operatorname{dim} W_{M}(X, S) \leq \delta-\gamma-1
$$

and hence

$$
\operatorname{dim} W \leq \operatorname{dim} \rho(W)+\operatorname{dim} W_{M}(X, S) \leq \sum_{1}^{\gamma} g_{i}+\delta-\gamma-1=g-2 .
$$

Remark 3.3. From now on we shall assume $\operatorname{dim} \rho(W)=\sum_{1}^{\gamma} g_{i}-1$.

Denote $\pi_{i}: \prod_{i=1}^{\gamma} \operatorname{Pic}^{d_{i}} C_{i} \rightarrow \operatorname{Pic}^{d_{i}} C_{i}$ the projection and $\rho_{i}:=\pi_{i} \circ \rho$

$$
\rho_{i}: W \longrightarrow \rho(W) \longrightarrow \rho_{i}(W) \subset \operatorname{Pic}^{d_{i}} C_{i} \text {. }
$$

As $\operatorname{dim} \prod_{i=1}^{\gamma} \operatorname{Pic}^{d_{i}} C_{i}=\sum_{1}^{\gamma} g_{i}$ and $\operatorname{dim} \rho(W)=\sum_{1}^{\gamma} g_{i}-1$, we get

$$
\operatorname{dim} \rho_{i}(W) \geq g_{i}-1, \quad \forall i
$$

and there can be at most most one index $i$ for which $\operatorname{dim} \rho_{i}(W)=g_{i}-1$

Case III. $\operatorname{dim} \rho(W)=\sum_{1}^{\gamma} g_{i}-1$ and $h^{0}(Y, M) \geq \delta+1$.

We claim that we can apply 2.11 to the general $M \in \rho(W)$, so that

$$
\operatorname{dim} W_{M}(X, S) \leq \delta-\gamma
$$

and we can conclude as follows:

$$
\operatorname{dim} W \leq \operatorname{dim} \rho(W)+\operatorname{dim} W_{M}(X, S) \leq g_{Y}-1+\delta-1=g-2 .
$$

To prove that the hypotheses of 2.11 hold, observe that (27) follows from the fact that $\underline{d}$ is stable (see the proof of 2.13). To prove the remaining assumption we argue by contradiction. Assume that for some admissible divisor $E$ with $\operatorname{Supp} E \subset \nu^{-1}(S)$ and $e:=\operatorname{deg} E$ we have

$$
h^{0}(Y, M(-E)) \geq h^{0}(Y, M)-e+1
$$

for $M$ general in $\rho(W)$. As $Y$ is the disjoint union of the $C_{i}$, we get

$$
h^{0}(Y, M(-E))=\sum_{i=1}^{\gamma} h^{0}\left(C_{i}, M_{i}\left(-E_{i}\right)\right) \geq \sum_{i=1}^{\gamma}\left(h^{0}\left(C_{i}, M_{i}\right)-e_{i}\right)+1
$$

where $E_{i}=E_{\mid C_{i}}, e_{i}:=\operatorname{deg} E_{i}$ and $M_{i}=M_{\mid C_{i}}$. Therefore there exists at least one index, say $i=1$, such that

$$
h^{0}\left(C_{1}, M_{1}(-E)\right) \geq h^{0}\left(C_{1}, M_{1}\right)-e_{1}+1 .
$$

The fact that $E$ is admissible implies that $e_{1} \leq h^{0}\left(C_{1}, M_{1}\right)$. Now, as $d_{1} \geq g_{1}$, there are two possiblities:

(a) $h^{0}\left(C_{1}, M_{1}\right)=d_{1}-g_{1}+1$

(b) $h^{0}\left(C_{1}, M_{1}\right) \geq d_{1}-g_{1}+2$

If (a) occurs, $\rho_{1}: W \rightarrow \mathrm{Pic}^{d_{1}} C_{1}$ is dominant. In fact, by the assumption $h^{0}(M) \geq \delta+1$, there exists an index $i \neq 1($ say $i=2)$ such that $h^{0}\left(C_{2}, M_{2}\right) \geq$ $d_{2}-g_{2}+2$, i.e. such that $M_{2}$ is a special line bundle on $C_{2}$. Therefore $\rho_{2}(W)$ cannot be dense in $\mathrm{Pic}^{d_{2}} C_{2}$. By 3.3, $\rho_{1}(W)$ is dense in $\mathrm{Pic}^{d_{1}} C_{1}$. Therefore we can apply Lemma 2.9 (with $Y=X=C_{1}$ and $\underline{d}=d_{1}$ ), getting that $E_{1}$ imposes independent conditions on $M_{1}$, a contradiction with (45). 
In case (b) we can assume $e_{1}=h^{0}\left(C_{1}, M_{1}\right) \geq d_{1}-g_{1}+2$. So $M_{1}$ is not a general point in $\operatorname{Pic}^{d_{1}} C_{1}$; by 3.3. $\operatorname{dim} \rho_{1}(W)=g_{1}-1$. Now (45) is $h^{0}\left(C_{1}, M_{1}\left(-E_{1}\right) \geq 1\right.$. Consider the map

$$
\begin{array}{ccc}
u_{E_{1}}: W_{d_{1}-e_{1}}^{0}\left(C_{1}\right) & \longrightarrow & \operatorname{Pic}^{d_{1}} C_{1} \\
N & \mapsto & N\left(+E_{1}\right)
\end{array}
$$

By what we said, $\operatorname{Im} u_{E_{1}}$ dominates $\rho_{1}(W)$, hence the variety $W_{d_{1}-e_{1}}^{0}\left(C_{1}\right)$ has dimension at least $g_{1}-1$. This is impossible, since (by (8))

$$
\operatorname{dim} W_{d_{1}-e_{1}}^{0}\left(C_{1}\right) \leq \min \left\{d_{1}-e_{1}, g_{1}\right\} \leq \min \left\{d_{1}-\left(d_{1}-g_{1}+2\right), g_{1}\right\}=g_{1}-2
$$

Case IV. $\operatorname{dim} \rho(W)=\sum_{1}^{\gamma} g_{i}-1$ and $h^{0}(Y, M)=\delta$.

If 2.11 applies, then we can argue as for Case 2 and we are done. Observe that if, for every $i=1, \ldots, \gamma$, every divisor $E_{i} \in \mathcal{A}\left(M_{i}, \nu^{-1}(S) \cap C_{i}\right)$ (cf. 2.7 (C)) imposes independent conditions on $M_{i}$, then every $E \in \mathcal{A}\left(M, \nu^{-1}(S)\right)$ imposes independent conditions on $M$ (indeed, arguing as in Case III

$\left.h^{0}(Y, M(-E))=\sum h^{0}\left(C_{i}, M_{i}\left(-E_{i}\right)\right)=\sum\left(h^{0}\left(C_{i}, M_{i}\right)-e_{i}\right)=h^{0}(Y, M)-e.\right)$

In such a case, 2.11 applies and we are done.

By 3.3 there are two possibilities.

(a) $\rho_{i}(W)$ is dense in $\operatorname{Pic}^{d_{i}} C_{i}$ for every $i$.

(b) There exists a unique index, say $i=1$ such that $\operatorname{dim} \rho_{1}(W)=g_{1}-1$, whereas for $i \geq 2 \rho_{i}(W)$ is dense.

In case (a), $M_{i}$ is general in $\mathrm{Pic}^{d_{i}} C_{i}$, hence by 2.9 and by what we observed above we can use 2.11 and we are done.

Now case (b) assuming that 2.11 cannot be applied. Let $E:=\sum_{j=1}^{\delta} q_{h(j)}^{j}$ be an admissible divisor for $M$ of the type constructed in 2.10 (with the same notation). If $E$ imposes independent conditions, i.e. if $h^{0}(Y, M(-E))=$ $h^{0}(M)-\delta=0$, we can apply 2.1 (iii) and obtain that $\operatorname{dim} W_{M}(X)=\gamma-\delta$. This is enough to conclude:

$$
\operatorname{dim} W \leq \operatorname{dim} \rho(W)+\operatorname{dim} W_{M}(X)=\sum_{1}^{\gamma} g_{i}-1+\delta-\gamma=g-2 .
$$

So, assume that $h^{0}(Y, M(-E)) \geq 1$. We have that $h^{0}\left(C_{i}, M_{i}\left(-E_{i}\right)\right)=0$ if $i \geq 2$, whereas $h^{0}\left(C_{1}, M_{1}\left(-E_{1}\right)\right) \geq 1$. Let us look at $M_{1}$ more closely. Using the fact that $M_{1}$ varies in a set of dimension $g_{1}-1$, one easily sees that

$$
h^{0}\left(C_{1}, M_{1}\left(-E_{1}\right)\right)=1 .
$$

It is trivial to check that we can assume, for a suitable $q \in \operatorname{Supp} E_{1}$, that $E_{1}=E_{1}^{\prime}+q$ with $E_{1}^{\prime}$ imposing independent conditions on $M_{1}$, i.e.

$$
h^{0}\left(C_{1}, M_{1}\left(-E_{1}^{\prime}\right)\right)=1
$$

so that $q$ is a base point of $M_{1}\left(-E_{1}^{\prime}\right)$.

Therefore

$$
h^{0}(Y, M(-E))=1
$$

and there exists a point $q \in E_{1}$ such that, setting $E^{\prime}=E-q_{1}$, the divisor $E^{\prime}$ imposes independent conditions on $M$. Now let $n$ be the node of $X$ of which 
the point $q_{1}$ is a branch, let $S^{\prime}=S \backslash n$; thus $E^{\prime}$ is supported on $\nu^{-1}\left(S^{\prime}\right)$. Let $\nu_{n}: X^{\prime} \rightarrow X$ be the normalization of $X$ at $n$, so that we can factor $\nu$

$$
Y \stackrel{\nu^{\prime}}{\longrightarrow} X^{\prime} \stackrel{\nu_{n}}{\longrightarrow} X
$$

and $\nu^{\prime}$ is the normalization of $X^{\prime}$. Of course, $X^{\prime}$ has $\delta^{\prime}=\delta-1$ nodes and $h^{0}(Y, M)=\delta^{\prime}+1$. As $E^{\prime}$ imposes independent conditions on $M$, we can apply 2.1 with respect to $\nu^{\prime}: Y \longrightarrow X^{\prime}$. This gives us that $W_{M}\left(X^{\prime}\right)=$ $F_{M}\left(X^{\prime}\right)$ and, for a general $L^{\prime} \in W_{M}\left(X^{\prime}\right)$

$$
h^{0}\left(X^{\prime}, L^{\prime}\right)=h^{0}(Y, M)-\delta^{\prime}=1 .
$$

Consider the following diagram

$$
\begin{array}{lcccc}
\operatorname{Pic} X & \stackrel{\nu_{n}^{*}}{\longrightarrow} & \operatorname{Pic} X^{\prime} & \stackrel{\left(\nu^{\prime}\right)^{*}}{\longrightarrow} & \operatorname{Pic} Y \\
W_{M}(X) & \longrightarrow & F_{M}\left(X^{\prime}\right) & \longrightarrow & M
\end{array}
$$

Observe that $n$ is not a separating node of $X$ (otherwise, by [1.8, $\Sigma(X)$ is empty and there is nothing to prove). Hence $\nu_{n}^{*}$ is a $k^{*}$-fibration and

$$
\operatorname{dim} F_{M}\left(X^{\prime}\right)=\delta^{\prime}-\gamma+1=\delta-\gamma .
$$

We now claim that the fiber $W_{L^{\prime}}(X)$ of $W_{M}(X)$ over the general point $L^{\prime} \in$ $F_{M}\left(X^{\prime}\right)$ has dimension $\leq 0$. By (48) we are in the situation of Lemma 2.5. which tells us that the only case when $\operatorname{dim} W_{L^{\prime}}(X)=1$ is when $L^{\prime}$ has a base point in each of two branches of $n$. Now this does not happen. Indeed, if $i \geq 2 M_{i}$ is general and hence has no base point over $X_{\text {sing; }}$; on the other hand $M_{1}$ varies in a codimension 1 subset of $\mathrm{Pic}^{d_{1}} C_{1}$ hence it has at most one base point over $X_{\text {sing }}$; therefore we can apply Lemma 1.4.

Concluding: $\operatorname{dim} W_{M}(X) \leq \delta-\gamma$. Arguing as in (47) we are done.

Example 3.4. The Theorem fails if we assume $\underline{d}$ semistable. The simplest case of $\underline{d} \in \Sigma^{s s}(X)$ with $W_{\underline{d}}(X)$ reducible is that of a curve of compact type, $X=C_{1} \cup C_{2}$, where $\bar{C}_{i}$ is smooth of genus $g_{i}$, $\# C_{1} \cap C_{2}=1$ and $\underline{d}=\left(g_{1}-1, g_{2}\right) ;$ (note that $\underline{d}$ is strictly semistable by 1.8$)$. Then

$$
W_{\underline{d}}(X)=\left(W_{g_{1}-1}\left(C_{1}\right) \times \operatorname{Pic}^{g_{2}} C_{2}\right) \cup\left(\operatorname{Pic}^{g_{1}-1} C_{1} \times \Theta_{q_{2}}\left(C_{2}\right)\right)
$$

where $q_{2} \in C_{2}$ is the point over the node and

$$
\Theta_{q_{2}}\left(C_{2}\right):=\left\{L \in \operatorname{Pic}^{g_{2}} C_{2}: h^{0}\left(C_{2}, L\left(-q_{2}\right)\right) \neq 0\right\} .
$$

Example 3.5. Here is an example where $\Sigma(X) \neq \emptyset$. Let $X=C_{1} \cup C_{2}$, with $C_{i}$ is smooth of genus $g_{i}$ and $\# C_{1} \cap C_{2}=2$, so that $g-1=g_{1}+g_{2}$. Set $\underline{d}=\left(g_{1}-1, g_{2}+1\right)$; note that $\Sigma(X)=\left\{\left(g_{1}, g_{2}\right)\right\}$ so that $\underline{d}$ is strictly semistable. Let $\nu: Y=C_{1} \amalg C_{2} \rightarrow X$ be the normalization and

$$
\operatorname{Pic}^{\underline{d}} X \stackrel{\nu^{*}}{\rightarrow} \operatorname{Pic}^{\underline{d}} Y=\operatorname{Pic}^{g_{1}-1} C_{1} \times \operatorname{Pic}^{g_{2}+1} C_{2}
$$

the pull-back map. Pick $M=\left(L_{1}, L_{2}\right) \in \operatorname{Pic} \underline{d} Y$. If $h^{0}\left(C_{1}, L_{1}\right) \neq 0$ then $h^{0}(Y, M) \geq 3$ and hence $F_{M}(X)=W_{M}(X)$. Therefore $W_{\underline{d}}(X)$ contains an irreducible component $V_{1}$

$$
V_{1}=\left(\nu^{*}\right)^{-1}\left(W_{g_{1}-1}\left(C_{1}\right) \times \mathrm{Pic}^{g_{2}+1} C_{2}\right) .
$$


Now suppose $h^{0}\left(C_{1}, L_{1}\right)=0$; if $h^{0}\left(C_{2}, L_{2}\left(-q_{2}-p_{2}\right)=0\right.$ (where $q_{2}$ and $p_{2}$ are the branches of $C_{2}$ over the two nodes) then $W_{M}(X)=\emptyset$. Conversely, if $h^{0}\left(C_{2}, L_{2}\left(-q_{2}-p_{2}\right) \neq 0\right.$, then $F_{M}(X)=W_{M}(X)$. Denote

$$
Z:=\left\{L_{2} \in \mathrm{Pic}^{g_{2}+1} C_{2}: h^{0}\left(C_{2}, L_{2}\left(-q_{2}-p_{2}\right) \neq 0\right\}\right.
$$

(it is easy to check that $Z$ is irreducible of dimension $g_{2}-1$ ). Then $W_{\underline{d}}(X)$ contains a second irreducible component $V_{2}$ where

$$
V_{2}=\left(\nu^{*}\right)^{-1}\left(\mathrm{Pic}^{g_{1}-1} C_{1} \times Z\right)
$$

So, $W_{\underline{d}}(X)=V_{1} \cup V_{2}$; note that, obviously, $V_{1}=A_{\underline{d}}(X)$.

\subsection{Dimension of the image of the Abel map.}

Proposition 3.6. Let $X$ be a curve of genus $g \geq 2$. Let $\underline{d} \in \mathbb{Z}^{\gamma}$ be a non-negative multidegree such that $|\underline{d}|=g-1$. Il $\underline{d}$ is semistable, then

(a) the general $L \in A_{\underline{d}}(X)$ satisfies $h^{0}(X, L)=1$;

(b) $\operatorname{dim} A_{\underline{d}}(X)=g-\overline{1}$.

Conversely, if $\underline{d}$ is not semistable, then

(A) for every $L \in A_{\underline{d}}(X)$ we have $h^{0}(X, L) \geq 2$;

(B) $\operatorname{dim} A_{\underline{d}}(X) \leq g-2$.

Proof. If $\underline{d}$ is stable, by Theorem 3.2 we know that $A_{\underline{d}}(X)=W_{\underline{d}}(X)$, $\operatorname{dim} A_{\underline{d}}(X)=g-1$ (by 1.10) and that the general point $L \in A_{\underline{d}}(X)$ has $h^{0}(X, L)=1$. So, for the first half of the statement, we need to consider the case where $X$ is reducible and $\underline{d}$ semistable but not stable. Thus, there exists a decomposition $X=V \cup \bar{Z}$, where $V$ and $Z$ are subcurves of respective arithmetic genus $g_{V}$ and $g_{Z}$, such that $V$ is connected,

$$
d_{V}=g_{V}-1 \text { and } d_{Z}=g_{Z}+\delta_{S}-1,
$$

where $S:=V \cap Z$ and $\delta_{S}:=\# S$.

Observe that, since $\underline{d} \geq 0$ we get that $g_{V} \geq 1$. By (2) we have

$$
g=g_{V}+g_{Z}+\delta_{S}-1 .
$$

Let $L$ be a general point in $A_{\underline{d}}(X)$; we can assume that $L$ is a line bundle on $X$ of type $L=\mathcal{O}_{X}(D)$ where $D$ is an effective divisor of multidegree $\underline{d}$ supported on the smooth locus of $X$. Consider the restrictions $L_{V}$ and $L_{Z}$ of $L$ to $V$ and $Z$; we have $h^{0}\left(V, L_{V}\right) \geq 1$. On the other hand $h^{0}\left(Z, L_{Z}\right) \geq$ $d_{Z}-g_{Z}+1=\delta_{S}$ (by Riemann-Roch and (50) ); moreover equality holds for a general $L_{Z} \in \operatorname{Pic}^{-} Z Z$, by Corollary 2.3 . Denote the partial normalization of $X$ at $S$ by

$$
\nu_{S}: Y_{S}=V \coprod Z \longrightarrow X
$$

and note that $\operatorname{Pic}^{\underline{d}} Y_{S}=\operatorname{Pic}^{\underline{d}} V \times \operatorname{Pic}^{\underline{d}} z Z$. Set $M=\nu_{S}^{*} L=\left(L_{V}, L_{Z}\right)$. Then for $L$ general

$$
h^{0}\left(Y_{S}, M\right)=h^{0}\left(V, L_{V}\right)+h^{0}\left(Z, L_{Z}\right)=\delta_{S}+1
$$

hence by Proposition 2.1 (15), which we can apply by Lemma 2.2(ii), we obtain that $h^{0}(X, L)=h^{0}\left(Y_{S}, M\right)-\delta_{S}=1$.

Now we compute $\operatorname{dim} A_{\underline{d}}(X)$ using induction on the number of irreducible components of $X$. The case $X$ irreducible has already been settled. Assume 
$X$ reducible; by what we said above, the pull back map $\nu_{S}^{*}$ restricted to $A_{\underline{d}}(X)$ gives a dominant rational map (denoted by $\rho$ )

$$
A_{\underline{d}}(X) \stackrel{\rho}{\longrightarrow} A_{\underline{d}_{V}}(V) \times \operatorname{Pic}^{\underline{d}} Z \text {. }
$$

Now recall that $\left|\underline{d}_{V}\right|=g_{V}-1 \geq 0$ by (50) and $\underline{d}_{V}$ is semistable on $V$ because $\underline{d}$ is semistable on $X$ (cf. 1.6). Furthermore $V$ has fewer components than $X$, hence we can use induction to conclude that $\operatorname{dim} A_{\underline{d}_{V}}(V)=d_{V}=g_{V}-1$.

If $M$ is a general point in the image of the above map $\rho$, then by (52) and 2.1 (iii), we see that $W_{M}(X)=F_{M}(X)$. We claim that $W_{M}(X) \subset A_{\underline{d}}(X)$. Indeed recall that $M=\nu_{S}^{*} \mathcal{O}_{X}(D)$ with $\operatorname{Supp} D \subset \dot{X}$, hence there exists an $L \in W_{M}(X)$ (namely, $L=\mathcal{O}_{X}(D)$ ) admitting a section that does not vanish at any node of $X$. Therefore the same holds for every line bundle in a dense open subset of $W_{M}(X)$ (which is irreducible, being equal to $F_{M}(X)$ ). This shows that $W_{M}(X) \subset A_{\underline{d}}(X)$. Therefore $\rho^{-1}(M)=F_{M}(X)$ and

$$
\operatorname{dim} A_{\underline{d}}(X)=g_{V}-1+g_{Z}+\delta_{S}-\gamma_{S}+1=g-1 .
$$

Conversely assume that $\underline{d}$ is not semistable. Then there exists a decomposition $X=V \cup Z$, where (as before) $V$ and $Z$ are subcurves of genus $g_{V}$ and $g_{Z}$ such that

$$
d_{V} \leq g_{V}-2 \text { and } d_{Z} \geq g_{Z}+\delta_{S}
$$

where $S:=V \cap Z$ and $\delta_{S}:=\# S$. Notice that $g_{V} \geq 2($ as $\underline{d} \geq 0)$.

We use the same notation as before. Let $L$ be a general point in $A_{\underline{d}}(X)$, so $L$ is of type $L=\mathcal{O}_{X}(D)$ with $D \geq 0$ supported on $\dot{X}$. We have $h^{0}\left(V, \underline{L}_{V}\right) \geq 1$ and $h^{0}\left(Z, L_{Z}\right) \geq d_{Z}-g_{Z}+1 \geq \delta_{S}+1$.

Consider $\nu_{S}: Y_{S}=V \amalg Z \longrightarrow X$ and set $M=\nu_{S}^{*} L=\left(L_{V}, L_{Z}\right)$. We have

$$
h^{0}\left(Y_{S}, M\right)=h^{0}\left(V, L_{V}\right)+h^{0}\left(Z, L_{Z}\right) \geq \delta_{S}+2
$$

hence by $2.1(13)$

$$
h^{0}(X, L) \geq 2
$$

proving part (A). To compute $\operatorname{dim} A_{\underline{d}}(X)$ consider again the rational map

$$
A_{\underline{d}}(X) \stackrel{\rho}{\longrightarrow} A_{\underline{d}_{V}}(V) \times \operatorname{Pic}^{\underline{d}} Z Z
$$

Since $\operatorname{dim} A_{\underline{d}_{V}}(V) \leq d_{V}$ (by Lemma 1.5) we get

$$
\operatorname{dim} A_{\underline{d}}(X) \leq d_{V}+g_{Z}+\operatorname{dim} W_{M}(X) \leq g_{V}-2+g_{Z}+\operatorname{dim} W_{M}(X)
$$

using (53) for the last inequality. Thus

$$
\operatorname{dim} A_{\underline{d}}(X) \leq g_{V}-2+g_{Z}+\delta_{S}-\gamma_{S}+1=g-2 .
$$

This proves $(\bar{B})$ and we are done.

3.2.1. Observe that, if $\underline{d}$ is not semistable, by (54) and 2.1 (13) we get $W_{M}(X)=F_{M}(X)$ and hence $\operatorname{dim} W_{M}(X)=\delta_{S}-\gamma_{S}+1$. So we can actually compute $\operatorname{dim} A_{\underline{d}}(X)$ more exactly, if necessary. For example, if $X=C_{1} \cup C_{2}$ and (say) $d_{1}=g_{1}-1-f(\underline{d}) \quad(f(\underline{d}) \geq 1$ measures the failure of semistability $\underline{d})$, we actually proved that

$$
\operatorname{dim} A_{\underline{d}}(X)=g-1-f(\underline{d}) .
$$

So, the farther is $\underline{d}$ from semistable, the smaller the dimension of $A_{\underline{d}}(X)$. 
Corollary 3.7. Let $R \subset X$ be a finite set of nonsingular points of $X$ and $\underline{d} \in \Sigma^{s s}(X)$. Then the general $L \in A_{\underline{d}}(X)$ has no base point in $R$.

Proof. It obviously suffices to assume $\# R=1$, so let $R=\{q\}$. If $L$ is general in $A_{\underline{d}}(X)$ we can assume that $L \in \operatorname{Im} \alpha \frac{d}{X}$. Set $L^{\prime}=L(-q)$ and $\underline{d}^{\prime}:=\underline{\operatorname{deg}} L^{\prime}$. If $q$ is a base point of $L$, then $L^{\prime} \in \operatorname{Im} \alpha \frac{d^{\prime}}{X}$. Therefore, if the general $L \in A_{\underline{d}}(X)$ has a base point in $q$, the map

$$
\begin{array}{rlr}
\operatorname{Im} \alpha \frac{d^{\prime}}{X} & \longrightarrow & A_{\frac{d}{d}}(X) \\
L^{\prime} & \mapsto
\end{array}
$$

must be dominant. But this is not possible, as $\operatorname{dim} A_{\underline{d}}(X)=g-1$ by 3.6. whereas obviously $\operatorname{dim} \operatorname{Im} \alpha \frac{d^{\prime}}{X} \leq\left|\underline{d}^{\prime}\right|=g-2$.

\section{Compactifying the theta Divisor}

4.0.2. Let $X$ be a connected nodal curve, $S \subset X_{\text {sing }}$, recall that $\delta_{S}:=\# S$ and $\nu_{S}: Y_{S} \rightarrow X$ is the normalization of $X$ at exactly the nodes in $S$. Let

$$
\widehat{X}_{S}=Y_{S} \cup_{i=1}^{\delta_{S}} E_{i}
$$

be the connected, nodal curve obtained by "blowing up" $X$ at $S$, so that $E_{i} \cong \mathbb{P}^{1}, \forall i$ and $E_{i}$ is called an exceptional component of $\widehat{X}_{S}$. We shall denote by $\widehat{M}$ a line bundle on $\widehat{X}_{S}$ and by $M \in \operatorname{Pic} Y_{S}$ its restriction to $Y_{S}$.

\subsection{The compactified Picard variety.}

4.1.1. The compactified Picard variety, or compactified jacobian, $\overline{P_{X}^{g-1}}$, in degree $g-1$, parametrizes equivalence classes of stable line bundles of degree $g-1$ on the curves $\widehat{X}_{S}$ as $S$ varies among all subsets of $X_{\text {sing. }}$.

Let us define stable line bundles and the equivalence relation among them. For every $S \subset X_{\text {sing }}$ consider the blow up of $X$ at $S, \widehat{X}_{S}=Y_{S} \cup_{i=1}^{\delta_{S}} E_{i}$ (cf. (56)). A stable line bundle $\widehat{M} \in \operatorname{Pic}^{d} \widehat{X}_{S}$ is such that, setting $M:=\widehat{M}_{Y_{S}}$, properties (1) and (2) below hold:

(1) $\underline{\operatorname{deg}} M \in \Sigma\left(Y_{S}\right)$ (i.e $\underline{\operatorname{deg}} M$ is stable on $Y_{S}$ )

(2) $\operatorname{deg}_{E_{i}} \widehat{M}=1$ for $i=1, \ldots \delta_{S}$.

We call a line bundle $\widehat{M} \in \operatorname{Pic}^{d} \widehat{X}_{S}$ semistable if it satisfies (2) and (1')

$\left(1^{\prime}\right) \quad \underline{\operatorname{deg}} M \in \Sigma^{s s}\left(Y_{S}\right)$.

These conditions determine only a line bundle on the normalization $\widehat{X}_{S}^{\nu}$ of $\widehat{X}_{S}$. But this is enough to describe the geometrical meaning of $\overline{P_{X}^{g-1}}$, since two stable line bundles $\widehat{M}$ and $\widehat{M}^{\prime}$ on $\widehat{X}_{S}$ are defined to be equivalent iff their pull backs to $\widehat{X}_{S}^{\nu}$ coincide (i.e. if $M$ and $M^{\prime}$ coincide on $Y_{S}$ ). 
4.1.2. Although $\overline{P_{X}^{g-1}}$ is constructed as a GIT-quotient, our terminology "stable/semistable line bundles" does not precisely reflect the GIT- stability/semistability. More precisely, denote $q_{X}: H_{X} \rightarrow \overline{P_{X}^{g-1}}$ the GIT quotient defining $\overline{P_{X}^{g-1}}$ (so that $H_{X}$ is a closed subset in the GIT-semistable locus of some Hilbert scheme). Then $H_{X}$ contains strictly GIT-semistable points, unless $X$ is irreducible. Let $\lambda \in \overline{P_{X}^{g-1}}$ be the image point of some strictly GIT-semistable point/orbit. Then $\lambda$ parametrizes several strictly GIT-semistable orbits, having pairwise intersecting closures. Hence there exists a unique orbit (of smallest dimension) in $q^{-1}(\lambda)$, which is closed in $H_{X}$. Of course, this orbit is strictly GIT-semistable, but the line bundle $\widehat{M} \in \operatorname{Pic} \widehat{X}$ corresponding to it will be stable according to our definition.

On the other hand, if $\lambda$ is the image point of a GIT-stable orbit, then the corresponding line bundle is stable under our definition.

Summarizing: our definition of stable line bundles is equivalent to GITsemistable with closed orbit in the semistable locus.

4.1.3. As we said, the points in $\overline{P_{X}^{g-1}}$ are in one-to-one correspondence with equivalence classes of stable line bundles. Any such class is uniquely determined by $S$ and by $M \in \operatorname{Pic} Y_{S}$ (provided that $\Sigma\left(Y_{S}\right)$ is not empty, of course), therefore the points of $\overline{P_{X}^{g-1}}$ will be denoted by pairs $[M, S]$, where $S \subset X_{\text {sing }}$ and $M \in \operatorname{Pic}^{\underline{d}} Y_{S}$ with $\underline{d} \in \Sigma\left(Y_{S}\right)$.

4.1.4. For technical reasons we need to consider semistable multidegrees that are not stable. Let $\underline{d} \in \Sigma^{s s}\left(Y_{S}\right)$ be a semistable multidegree of $Y_{S}$; a node $n$ of $Y_{S}$ is called destabilizing for $\underline{d}$ if there exists a connected subcurve $Z \subset Y_{S}$ such that $n \in Z \cap Z^{C}$ and $d_{Z}=p_{a}(Z)-1\left(Z^{C}=\overline{Y \backslash Z}\right)$. We set

$$
S(\underline{d}):=\left\{n \in\left(Y_{S}\right)_{\text {sing }}: n \text { is destabilizing for } \underline{d}\right\}
$$

Observe that

$$
S(\underline{d})=\emptyset \Leftrightarrow \underline{d} \in \Sigma\left(Y_{S}\right) .
$$

We call $Y_{S}(\underline{d})$ the normalization of $Y_{S}$ at $S(\underline{d})$, so that we have

$$
Y_{S}(\underline{d})=Y_{S \cup S(\underline{d})} \stackrel{\nu_{d}}{\longrightarrow} Y_{S} \stackrel{\nu_{S}}{\longrightarrow} X
$$

where $\nu_{\underline{d}}$ is the normalization map.

Assume that $\underline{d}$ is strictly semistable, i.e. $S(\underline{d})$ is not empty. Then the dual graph of $Y_{S}$ has an orientation such that for every subcurve $Z \subset Y_{S}$ such that $d_{Z}=p_{a}(Z)-1$, all the edges between $\Gamma_{Z}$ and $\Gamma_{Z^{C}}$ go from $\Gamma_{Z}$ to $\Gamma_{Z^{C}}$ (by 1.3.2). Therefore, if we consider $Y_{S}(\underline{d})$ and use the convention of 1.7, for every destabilizing node $n \in Z \cap Z^{C}$, we have $q_{1}^{n} \in Z$ and $q_{2}^{n} \in Z^{C}$ (abusing notation by denoting $Z=\nu_{\underline{d}}^{-1}(Z)$ and $Z^{C}=\nu_{\underline{d}}^{-1}\left(Z^{C}\right)$ ). Denote

$$
T(\underline{d}):=\sum_{n \in S(\underline{d})} q_{2}^{n} \text { and } \underline{t}(\underline{d}):=\underline{\operatorname{deg}} T(\underline{d}) .
$$

By construction, $\underline{d}-\underline{t}(\underline{d})$ is a stable multidegree for $Y_{S}(\underline{d})$. Set

$$
\underline{d}^{s t}:=\underline{d}-\underline{t}(\underline{d}) \in \Sigma\left(Y_{S}(\underline{d})\right) .
$$


The following statement, summarizes various known facts about $\overline{P_{X}^{g-1}}$. The only novelty is that we use line bundles on the partial normalizations of $X$, rather than torsion free sheaves on $X$ (as in [AK80, OS79, S94 among others) or line bundles on the blow-ups of $X$ (as in C94).

Fact 4.1. $\overline{P_{X}^{g-1}}$ is a connected, reduced, projective scheme of pure dimension g. It has a stratification

$$
\overline{P_{X}^{g-1}}=\coprod_{\substack{\emptyset \subseteq S \subseteq X_{\text {sing }} \\ \underline{d} \in \Sigma\left(Y_{S}\right)}} P^{\frac{d}{S}}
$$

such that the following properties hold.

(i) For every $S \subset X_{\text {sing }}$ and every $\underline{d} \in \Sigma\left(Y_{S}\right)$ there is a canonical isomorphism (notation in 4.1.3)

$$
\begin{array}{ccr}
\operatorname{Pic} \underline{d} Y_{S} & \stackrel{\epsilon}{S} & P \frac{d}{S} \\
M & \mapsto & {[M, S]}
\end{array}
$$

In particular, if $P \frac{d}{S} \neq \emptyset$, then $\operatorname{dim} P \frac{d}{S}=g-\delta_{S}+\gamma_{S}-1$

(ii) (More generally) For every $S \subset X_{\text {sing }}$ and every $\underline{d} \in \Sigma^{s s}\left(Y_{S}\right)$ there is a

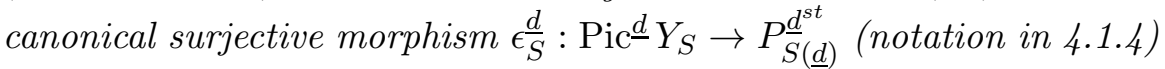
which factors as follows

$$
\begin{aligned}
& \epsilon \frac{d}{S}: \operatorname{Pic} \underline{d} Y_{S} \stackrel{\tau}{\longrightarrow} \quad \operatorname{Pic}^{\underline{d}^{s t}} Y_{S}(\underline{d}) \quad \stackrel{\epsilon^{\frac{d^{s t}}{S(d)}} \longrightarrow}{\longrightarrow} P \underline{d}^{s(\underline{d})} \\
& L \quad \mapsto \quad \nu_{\underline{d}}^{*} L \otimes \mathcal{O}_{Y_{S}(\underline{d})}\left(-\sum_{n \in S(\underline{d})} q_{2}^{n}\right)
\end{aligned}
$$

where $\tau$ is surjective with fibers $\left(k^{*}\right)^{b}, b=\delta_{S(\underline{d})}-\gamma_{S(\underline{d})}+1$, and $\left.\epsilon_{S}^{\underline{d}^{s t}}{ }^{\underline{d}}\right)$ is an isomorphism.

(iii) $P{\overline{d^{\prime}}}^{\prime} \subset \overline{P_{S}^{\underline{d}}}$ if and only if $S \subset S^{\prime}$ and $\underline{d} \geq \underline{d}^{\prime}$ (i.e. $\left.d_{i} \geq d_{i}^{\prime}, \forall i=1, \ldots, \gamma\right)$. In such a case, $\#\left(\left(S^{\prime} \backslash S\right) \cap \overline{C_{i}}\right)=d_{i}-d_{i}^{\prime}$ (recall that $\left.X=\cup_{1}^{\gamma} \overline{C_{i}}\right)$.

(iv) Denote $P_{X}^{g-1}$ the smooth locus of $\overline{P_{X}^{g-1}}$, then

$$
P_{X}^{g-1}=\coprod_{\underline{d} \in \Sigma(\widetilde{X})} P_{\bar{X}_{s e p}}^{\underline{d}} \cong \coprod_{\underline{d} \in \Sigma(\widetilde{X})} \operatorname{Pic}^{\underline{d}} \widetilde{X}
$$

where $\widetilde{X} \rightarrow X$ is the normalization at the separating nodes (cf. 1.9) and the isomorphism is the canonical one described in part (i).

4.2. The theta divisor of the compactified jacobian. We shall now define the theta divisor of $\overline{P_{X}^{g-1}}$ using the stratification given above. A natural thing to do is to consider the irreducible strata $P \frac{d}{S}$ of dimension $g$ of $\overline{P_{X}^{g-1}}$, consider $W_{\underline{d}}(X)$ in such strata and then take their closure. Recalling Lemma 1.8, we can easily list the $g$-dimensional strata: denote $X_{\text {sep }} \subset X_{\text {sing }}$ the set of separating nodes of $X$ and let $\widetilde{X} \rightarrow X$ be the normalization of $X$ at $X_{\text {sep }}$ (as in 4.1 (iv) ). Thus $\widetilde{X}$ is a nodal curve having $c=\# X_{\text {sep }}+1$ connected components. Finally, set $\widetilde{b}=\# \Sigma(\widetilde{X})$. We have 
Lemma - Definition 4.2. Let $X$ be a connected nodal curve. Using $\epsilon \frac{d}{S}$ of of 4.1 (2i) as an identification, we define the theta divisor $\Theta(X)$ of $\overline{P_{X}^{g-1}}$ as

$$
\left.\Theta(X):=\overline{\bigcup_{\underline{d} \in \Sigma(\widetilde{X})} W_{\underline{d}}(\widetilde{X})} \subset \overline{P_{X}^{g-1}}\right) .
$$

$\Theta(X)$ has $c \widetilde{b}$ irreducible components, all of dimension $g-1$.

Proof. If $X$ is free from separating nodes (i.e. $c=1$ ) the statement follows trivially from Theorem 3.2 . Otherwise, let $\widetilde{X}=X_{1} \amalg \ldots \amalg X_{c}$ be the decomposition into connected components. Then $g=\sum_{1}^{c} p_{a}\left(X_{i}\right)$ and

$$
W_{\underline{d}}(\widetilde{X})=\bigcup_{i=1}^{c}\left(W_{\underline{d}_{i}}\left(X_{i}\right) \times \prod_{\substack{j \neq i \\ j=1, \ldots, c}} \operatorname{Pic}^{\underline{d}_{j}} X_{j}\right)
$$

where $\underline{d}_{i}$ denotes the restriction of $\underline{d}$ to $X_{i}$. Since $X_{i}$ is connected and $\underline{d}_{i}$ is stable, $W_{\underline{d}_{i}}\left(X_{i}\right)$ is irreducible of dimension $p_{a}\left(X_{i}\right)-1$, hence we are done (cf. [1.9) .

Remark 4.3. Definition 4.2 is natural from the point of view of the present paper, nevertheless it does not coincide with the one given in [E97] or (which is the same) in [Al04]. From Theorem 4.5 one immediately derives that the definitions are the same. In particular our $\Theta(X)$ is Cartier and ample.

In the following simple Lemma (cf. [P07] 2.1 for an analogous statement) we use the notation in 4.0.2.

Lemma 4.4. Let $S \subset X_{\text {sing }}$ and $M \in \operatorname{Pic} Y_{S}$. Pick $\widehat{M} \in \operatorname{Pic} \widehat{X}_{S}$ such that $\widehat{M}_{\mid Y_{S}}=M$ and $\widehat{M}_{E}=\mathcal{O}_{E}(1)$ for every exceptional component $E$ of $\widehat{X}_{S}$. Then $h^{0}\left(\widehat{X}_{S}, \widehat{M}\right)=h^{0}\left(Y_{S}, M\right)$.

Proof. Obviously, for any pair of points $p_{1}, p_{2} \in \mathbb{P}^{1}$ and any pair $a_{1}, a_{2} \in k$ there exists a unique section $s \in H^{0}\left(\mathbb{P}^{1}, \mathcal{O}_{\mathbb{P}^{1}}(1)\right)$ such that $s\left(p_{1}\right)=a_{i}$ for $i=1,2$. So, every section $s_{Y} \in H^{0}(Y, M)$ extends to a unique section of $H^{0}\left(\widehat{X}_{S}, \widehat{M}\right)$ determined by $s_{Y}$ and by the gluing data defining $\widehat{M}$. Conversely of course any section in $H^{0}\left(\widehat{X}_{S}, \widehat{M}\right)$ restricts to a section of $M$.

Theorem 4.5. Let $X$ be a connected nodal curve. The stratification of $\overline{P_{X}^{g-1}}$ given by 4.1 induces the following canonical stratification:

(64) $\Theta(X)=\coprod_{\substack{\emptyset \subseteq S \subseteq X_{\text {sing }} \\ \underline{d} \in \Sigma\left(Y_{S}\right)}} \Theta \frac{d}{S}$, with canonical isomorphisms $\Theta \frac{d}{S} \cong W_{\underline{d}}\left(Y_{S}\right)$.

Equivalently

$$
\Theta(X)=\left\{[M, S] \in \overline{P_{X}^{g-1}}: h^{0}\left(\widehat{X}_{S}, \widehat{M}\right) \neq 0\right\} .
$$

Proof. The equivalence of the two descriptions follows immediately from Fact 4.1 and Lemma 4.4. Furthermore it is clear that

$$
\Theta(X) \subset\left\{[M, S] \in \overline{P_{X}^{g-1}}: h^{0}\left(\widehat{X}_{S}, \widehat{M}\right) \neq 0\right\}
$$

(by uppersemicontinuity of $h^{0}$ ). So we need to prove the other inclusion. 
Part 1: $\quad$ proof assuming $X$ free from separating nodes.

Recall that in this case

$$
\Theta(X)=\overline{\bigcup_{\underline{d} \in \Sigma(X)} W_{\underline{d}}(X)}
$$

We shall use Abel maps (see 1.2.3): recall that $\alpha \bar{Y}_{S}$ is the $\underline{d}$-th Abel map of $Y_{S}$ and the closure of its image in $\operatorname{Pic} \underline{\underline{d}} Y_{S}$ is denoted by $A_{\underline{d}}\left(Y_{S}\right)$.

Step 1. Assume $\# S=1$ and let $\underline{d} \in \Sigma^{s s}\left(Y_{S}\right)$. Then there exists $\underline{e} \in \Sigma(X)$ such that (using Theorem 3.2 for the equality below)

$$
\epsilon \frac{d}{S}\left(A_{\underline{d}}\left(Y_{S}\right)\right) \subset \overline{\epsilon_{\bar{\emptyset}}^{e}\left(W_{\underline{e}}(X)\right)}=\bar{\epsilon} \overline{\bar{\emptyset}}\left(A_{\underline{e}}(X)\right) .
$$

In particular, if $[M, S] \in \overline{P_{X}^{g-1}}$ (so that $\underline{\operatorname{deg}} M \in \Sigma\left(Y_{S}\right)$ ) satisfies $\# S=1$ and $h^{0}\left(\widehat{X}_{S}, \widehat{M}\right) \neq 0$, then $[M, S] \in \Theta(X)$.

Let $M \in \operatorname{Pic} \underline{d}\left(Y_{S}\right)$ with $M \in A_{\underline{d}}\left(Y_{S}\right)$ and $\underline{\operatorname{deg}} M=\underline{d} \in \Sigma^{s s}\left(Y_{S}\right)$. As $X$ is free from separating nodes, $Y_{S}$ is connected.

Observe that, by 4.1(iv), $\bar{P}_{X}^{g-1}$ is the closure of its open subset

$$
P_{X}^{g-1}=\coprod_{\underline{e} \in \Sigma(X)} P_{\bar{\emptyset}}^{\underline{e}} \cong \coprod_{\underline{e} \in \Sigma(X)} \operatorname{Pic}^{\underline{e}} X .
$$

Therefore there exists an $\underline{e} \in \Sigma(X)$ such that $\epsilon_{S}^{\frac{d}{S}}(M) \in \overline{P_{\bar{\emptyset}}^{\underline{e}}}=\overline{\operatorname{Pic}^{\underline{e}} X}$.

Since $\# S=1,|\underline{d}|=p_{a}\left(Y_{S}\right)-1=g-2=|\underline{e}|-1$. Furthermore $\underline{d} \leq \underline{e}$ (by 4.1 (iii)). Therefore there exists a unique index $i \in\{1, \ldots, \gamma\}$, say $i=1$, such that $d_{1}=e_{1}-1$ and $d_{i}=e_{i}$ for $i \geq 2$.

Set $S=\{n\}$, consider $\nu_{S}: Y_{S} \rightarrow X$ the normalization at $n$ and let $C_{1}$ be the first component of $Y_{S}$. Since $d_{1}=e_{1}-1$, by 4.1 (iii) $C_{1}$ contains one of the two branches of $n$, call $q_{1}$ this branch. Let now $p_{t} \in C_{1}$ be a moving point specializing to $q_{1}$.

We can assume that $M$ is a general point in $A_{\underline{d}}\left(Y_{S}\right)$ (which is irreducible of dimension $\left.p_{a}\left(Y_{S}\right)-1\right)$ in particular that $M$ is in the image of the Abel map, that $h^{0}\left(Y_{S}, M\right)=1$, and that $M$ has no base point lying over $n$ (by 3.6 and 3.7). Therefore there exists $L \in \operatorname{Pic} X$ such that $\nu_{S}^{*} L=M$ and

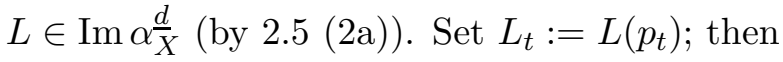

$$
\underline{\operatorname{deg}} L_{t}=\underline{d}+(1,0, \ldots, 0)=\underline{e} \in \Sigma(X)
$$

and $L_{t} \in \operatorname{Im} \alpha \frac{e}{X}$, in particular $h^{0}\left(X, L_{t}\right) \neq 0$. As $p_{t}$ specializes to $q_{1}$, we have that $\epsilon_{\bar{\emptyset}}^{e}\left(L_{t}\right)$ specializes to $\epsilon \frac{d}{S}(M)$ so we are done with Step 1.

Step 2. For every $S$ such that $\# S \geq 2$ and $\underline{d} \in \Sigma^{s s}\left(Y_{S}\right)$, there exist $S^{\prime} \subset S$ such that $\# S^{\prime}=\# S-1$, and a $\underline{d}^{\prime} \in \Sigma^{s s}\left(Y_{S^{\prime}}\right)$ such that

$$
\epsilon \bar{S}\left(A_{\underline{d}}\left(Y_{S}\right)\right) \subset \overline{\epsilon \frac{d^{\prime}}{S^{\prime}}\left(A_{\underline{d}^{\prime}}\left(Y_{S^{\prime}}\right)\right.} .
$$

Let $\underline{d}$ be a semistable multidegree for $Y_{S}$. Consider the dual graph $\Gamma_{Y_{S}}$ and an orientation on it inducing $\underline{d}$. Note that $\Gamma_{Y_{S}}$ is the subgraph of $\Gamma_{X}$ obtained by removing the edges corresponding to $S$. It is clear that if we 
add to $\Gamma_{Y_{S}}$ any edge $n$ of $\Gamma$, (so that $n \in S$ ) oriented however we like, we obtain a new oriented graph $\Gamma^{\prime}$ such that

$$
\Gamma_{Y_{S}} \subset \Gamma^{\prime} \subset \Gamma_{X}
$$

Set $S^{\prime}=S \backslash\{n\}$, thus $\Gamma^{\prime}$ is the dual graph of the curve $Y_{S^{\prime}}$ obtained by normalizing $X$ at $S^{\prime}$. Thus we have a map $Y_{S} \rightarrow Y_{S^{\prime}}$ which is the normalization of $Y_{S^{\prime}}$ at $n$.

The given orientation on $\Gamma^{\prime}$ corresponds to a semistable multidegree $\underline{d}^{\prime}$ such that $\left|\underline{d}^{\prime}\right|=|\underline{d}|+1$ and $\underline{d}^{\prime} \geq \underline{d}$.

From now on we can argue as for Step 1, with $Y_{S^{\prime}}$ playing the role of $X$. More precisely, if $Y_{S}$ is connected, then the argument is exactly the same: start from a general $M \in A_{\underline{d}}\left(Y_{S}\right)$ and construct a family of line bundles $L_{t}=L\left(p_{t}\right) \in A_{\underline{d}^{\prime}}\left(Y_{S^{\prime}}\right)$ such that $p_{t}$ is a smooth point of $Y_{S^{\prime}}$ specializing to $n$, and $L \in A_{\underline{d}}\left(Y_{S^{\prime}}\right)$ such that $L$ pulls back to $M$. Then $\epsilon_{S^{\prime}}\left(L_{t}\right)$ specializes to $\epsilon_{S} \frac{d}{S}(M)$.

If $Y_{S}$ is not connected, then the general $M \in A_{\underline{d}}\left(Y_{S}\right)$ has $h^{0}(M) \geq 2$, and it has no base point over $n$ (by 3.7). We now apply 2.6 to obtain $L \in \operatorname{Im} \alpha \frac{d}{Y_{S^{\prime}}}$ which pulls back to $M$. The rest of the argument is the same as before.

This conludes the proof of Step 2.

\section{Step 3. End of the proof of Part 1.}

To prove the theorem, we pick $[M, S] \in \overline{P_{X}^{g-1}}$ such that $M \in W_{\underline{d}}\left(Y_{S}\right)$; since $\underline{d}$ is stable, we have that $W_{\underline{d}}\left(Y_{S}\right)=A_{\underline{d}}\left(Y_{S}\right)$ by 3.2 (applied to every connected component of $Y_{S}$ ).

Using Step 2 we can decrease the cardinality of $S$ at the cost of passing from a stable multidegree to a semistable one (which is why the assumption for Step 1 is that $\underline{d}$ is semistable, rather than stable). Iterating Step 2 finitely many times, we reduce the proof of the theorem to Step 1. So the theorem is proved for $X$ free from separating nodes.

Part 2: proof assuming $X_{\text {sep }}$ not empty.

Recall that $\tilde{X} \rightarrow X$ is the normalization of $X$ at $X_{\text {sep }}$ and $\widetilde{X}=\cup_{i=1}^{c} X_{i}$ denotes the decomposition of $\tilde{X}$ into connected components; set $g_{i}=p_{a}\left(X_{i}\right)$. By fact 4.1 we have a canonical isomorphism

$$
\overline{P_{X}^{g-1}} \cong \prod_{i=1}^{c} \overline{P_{X_{i}}^{g_{i}-1}}
$$

and, by Definition 4.2, another canonical isomorphism

$$
\Theta(X) \cong \bigcup_{j=1}^{c}\left(\Theta\left(X_{j}\right) \times \prod_{\substack{i \neq j \\ 1 \leq i \leq c}} \overline{P_{X_{i}}^{g_{i}-1}}\right) .
$$

Let $[M, S] \in \overline{P_{X}^{g-1}}$ be such that $h^{0}\left(Y_{S}, M\right) \neq 0$. Now $S \supset X_{\text {sep }}$ hence we can factor

$$
\nu_{S}: Y_{S} \stackrel{\widetilde{\nu_{S}}}{\longrightarrow} \widetilde{X} \longrightarrow X
$$

and denote $Y_{i}={\widetilde{\nu_{S}}}^{-1}\left(X_{i}\right)$, so that $Y_{S}$ is the disjoint union of $Y_{1}, \ldots Y_{c}$. Note that $Y_{i}$ is the normalization of $X_{i}$ at a certain set of nodes, $S_{i}$, of $X_{i}$. 
Therefore, under the isomorphism (66), the point $[M, S]$ corresponds to the point $\left(\left[M_{1}, S_{1}\right], \ldots,\left[M_{c}, S_{c}\right]\right) \in \prod_{i=1}^{c} \overline{P_{X_{i}}^{g_{i}-1}}$ where $M_{i}=M_{Y_{i}}$.

Furthermore, $h^{0}\left(Y_{S}, M\right)=\sum_{1}^{c} h^{0}\left(Y_{i}, M_{i}\right)$, hence there exists an index, say $i=1$, such that $h^{0}\left(Y_{1}, M_{1}\right) \neq 0$. Now, $X_{1}$ is free from separating nodes, therefore by the first part of the proof we obtain

$$
\left[M_{1}, S_{1}\right] \in \Theta\left(X_{1}\right) .
$$

By (67), this implies $[M, S] \in \Theta(X)$ finishing the proof.

Example 4.6. Let $X=C_{1} \cup C_{2}$ with $\# C_{1} \cap C_{2}=1$; then $\Sigma(X)$ is empty, while $\Sigma(Y)=\left\{\left(g_{1}-1, g_{2}-1\right)\right\}$ where $Y$ is the normalization of $X$. The points of $\overline{P_{X}^{g-1}}$ correspond to line bundles of multidegree $\left(g_{1}-1, g_{2}-1\right)$ on $Y$ or to equivalence classes of line bundles on the curve $\widehat{X}$ obtained by blowing the unique node of $X$. More precisely, if we order the components of $\widehat{X}$ so that $\widehat{X}=C_{1} \cup E \cup C_{2}$ (where $E \cong \mathbb{P}^{1}$ ), then $\overline{P_{X}^{g-1}}$ bijectively parametrizes line bundles of multidegree $\left(g_{1}-1,1, g_{2}-1\right)$ on $\widehat{X}$. There is a canonical isomorphism $\overline{P_{X}^{g-1}} \cong \mathrm{Pic}^{g_{1}-1} C_{1} \times \mathrm{Pic}^{g_{2}-1} C_{2}$.

Now, $\Theta(X)$ is canonically isomorphic to $W_{\left(g_{1}-1, g_{2}-1\right)}(Y)$, which we can easily describe by means of 1.3 . We obtain three different cases.

Case 1: $g_{i} \neq 0 i=1,2$. Then $\Theta(X)$ has two irreducible components:

$$
\Theta(X)=\left(W_{g_{1}-1}\left(C_{1}\right) \times \mathrm{Pic}^{g_{2}-1} C_{2}\right) \cup\left(\mathrm{Pic}^{g_{1}-1} C_{1} \times W_{g_{2}-1}\left(C_{2}\right)\right) .
$$

Case 2: $g_{1}=0$ and $g_{2} \neq 0$. Then the first component in (68) is empty and we get $\Theta(X) \cong W_{g_{2}-1}\left(C_{2}\right) \cong \Theta\left(C_{2}\right)$.

Case 3: $g_{1}=g_{2}=0$. Then $\Theta(X)$ is empty.

Example 4.7. Let $X=C_{1} \cup C_{2}$ with $\# C_{1} \cap C_{2}=\delta \geq 2$. Then $g-1=$ $g_{1}+g_{2}+\delta-2$. We have

$$
\Sigma(X)=\left\{\left(g_{1}, g_{2}+\delta-2\right),\left(g_{1}+1, g_{2}+\delta-1\right), \ldots,\left(g_{1}+\delta-2, g_{2}\right)\right\}
$$

so that $\overline{P_{X}^{g-1}}$ has $\delta-1$ irreducible components of dimension $g$ and its smooth locus has a canonical isomorphism (cf. 4.1(iv))

$$
P_{X}^{g-1}=\coprod_{i=0}^{\delta-2} P_{\emptyset}^{\left(g_{1}+i, g_{2}+\delta-i-2\right)} \cong \coprod_{i=0}^{\delta-2} \operatorname{Pic}^{\left(g_{1}+i, g_{2}+\delta-i-2\right)} X .
$$

For every set $S \subset X_{\text {sing }}$ such that $\# S=k$ with $1 \leq k \leq \delta-2$, we have

$$
\Sigma\left(Y_{S}\right)=\left\{\left(g_{1}, g_{2}+\delta-k-2\right), \ldots,\left(g_{1}+\delta-k-2, g_{2}\right)\right\}
$$

so that $\overline{P_{X}^{g-1}}$ has a total of $(\delta-k-1)\left(\begin{array}{l}\delta \\ k\end{array}\right)$ strata of codimension $k$, each of which is isomorphic to $\operatorname{Pic}^{\underline{d}} Y_{S}$. If $k=\delta-1$ then for any choice of $\delta-1$ nodes, the curve obtained by blowing up $X$ at such nodes has a separating node, hence $\Sigma\left(Y_{S}\right)$ is empty. Finally the last stratum corresponds to $S=X_{\text {sing }}$ and $\underline{d}=\left(g_{1}-1, g_{2}-1\right)$ (the unique stable multidegree on $\left.C_{1} \amalg C_{2}\right)$ and has codimension $\delta-1$. We have

$$
P_{X_{\text {sing }}}^{\left(g_{1}-1, g_{2}-1\right)} \cong \mathrm{Pic}^{g_{1}-1} C_{1} \times \operatorname{Pic}^{g_{2}-1} C_{2} .
$$

Now let us describe $\Theta(X)$, showing that it has $\delta-1$ ireducible components. The stratification of $\Theta(X)$ contains $\delta-1$ strata of dimension $g-1$ all 
irreducible, one for every $g$-dimensional stratum of $\overline{P_{X}^{g-1}}$. Indeed for every $\underline{d} \in \Sigma(X)$ we have $\Theta \frac{d}{\emptyset} \cong W_{\underline{d}}(X)$ which is irreducible of dimension $g-1$, by Theorem 3.2 .

For every set $S \subset X_{\text {sing }}$ such that $\# S=k$ with $1 \leq k \leq \delta-2$, we have that $Y_{S}$ is connected and free from separating nodes, so that for every $\underline{d} \in \Sigma\left(Y_{S}\right)$ we get an irreducible stratum of dimension $g-k-1$ isomorphic to $W_{\underline{d}}\left(Y_{S}\right)$. If $k=\delta-1$ there are no strata (as before). If $k=\delta$ we get a stratum isomorhic to the theta divisor computed in Example 4.6 (cf. (68)).

\section{REFERENCES}

[A102] Alexeev, V.: Complete moduli in the presence of semiabelian group action. Ann. of Math. (2) 155 (2002), no. 3, 611-708.

[Al04] Alexeev, V.: Compactified Jacobians and Torelli map. Publ. RIMS, Kyoto Univ. 40 (2004), 1241-1265.

[AK80] Altman, A.; Kleiman, S.. Compactifying the Picard scheme. Adv. Math. 35 (1980), 50-112.

[ACGH] Arbarello, E.; Cornalba, M.; Griffiths, P. A.; Harris, J Geometry of algebraic curves. Vol. I. Grundlehren der Mathematischen Wissenschaften 267. SpringerVerlag, New York, 1985.

[B77] Beauville, A.: Prym varietes and the Schottky problem. Invent. Math. 41 (1977), no. 2, 149-196.

[C94] Caporaso, L. A compactification of the universal Picard variety over the moduli space of stable curves. J. of the Amer. Math. Soc. 7 (1994), 589-660.

[C05] Caporaso, L. Néron models and compactified Picard schemes over the moduli stack of stable curves. Amer. J. of Math., to appear. Also available at math.AG/0502171

[E97] Esteves, E.: Very ampleness for theta on the compactified Jacobian. Math. Z. 226 (1997), no. 2, 181-191.

[HM] Harris, J.; Morrison, I.:Moduli of curves. Graduate Texts in Mathematics, 187. Springer-Verlag, New York, 1998.

[OS79] Oda, T.;Seshadri, C.S.: Compactifications of the generalized Jacobian variety. Trans. A.M.S. 253 (1979) 1-90

[P07] Pacini, M.: Spin curves over non-stable curves. Comm. in Alg. to appear.

[S94] Simpson, C. T. : Moduli of representations of the fundamental group of a smooth projective variety. Inst. Hautes Études Sci. Publ. Math., 80: 5-79, 1994.

[S94] Soucaris, A. The ampleness of the theta divisor on the compactified Jacobian of a proper and integral curve. Compositio Math. 93 (1994), no. 3, 231-242.

Lucia Caporaso caporaso@mat.uniroma3.it

Dipartimento di Matematica, Università Roma Tre

Largo S. L. Murialdo 100146 Roma - Italy 